\title{
Microscopic self-consistent theory of Josephson junctions including dynamical electron correlations.
}

\author{
P. Miller and J. K. Freericks \\ Department of Physics, Georgetown University, \\ Washington, D.C. 20057-0995, U.S.A.
}

\begin{abstract}
We formulate a fully self-consistent, microscopic model to study the retardation and correlation effects of the barrier within a Josephson junction. The junction is described by a series of planes, with electronic correlation included through a local self energy for each plane. We calculate current-phase relationships for various junctions, which include non-magnetic impurities in the barrier region, or an interfacial scattering potential. Our results indicate that the linear response of the supercurrent to phase across the barrier region is a good, but not exact indicator of the critical current. Our calculations of the local density of states show the currentcarrying Andreev bound states and their energy evolution with the phase difference across the junction.

We calculate the figure of merit for a Josephson junction, which is the product of the critical current, $I_{c}$, and the normal state resistance, $R_{N}$, for junctions with different barrier materials. The normal state resistance is calculated using the Kubo formula, for a system with zero current flow and no superconducting order. Semiclassical calculations would predict that these two quantities are determined by the transmission probabilities of electrons in such a way that the product is constant for a given superconductor at fixed temperature. Our self-consistent solutions for different types of barrier indicate that this is not the case. We suggest some forms of barrier which could increase the $I_{c} R_{N}$ product, and hence improve the frequency response of a Josephson device.
\end{abstract}

Typeset using REVTEX 


\section{INTRODUCTION}

Some of the most successful and most promising electronics applications of superconductors involve Josephson junctions, where two superconducting regions are coupled through a barrier region, made from a non-superconducting material1. The drive to make electronic devices using Josephson junctions has been motivated by their naturally high operating frequencies, far in excess of the speeds obtainable in standard silicon technology. The operational frequency of an ideal Josephson junction using rapid single flux quantum (RSFQ) logic is $I_{c} R_{N} e / \hbar$, where $I_{c}$ is the critical current, and $R_{N}$ is the normal state resistance of the junction自目. Hence a maximum value of the product, $I_{c} R_{N}$ leads to optimal performance. At present, low temperature superconductors have been used to produce junctions with $I_{c} R_{N}$ products up to $1 \mathrm{mV}$, and operational speeds reaching 770 GHz 9 , while junctions of high-temperature superconductors have achieved $I_{c} R_{N}$ products reaching from $1 \mathrm{mV}$ to $\approx 20 \mathrm{mV}$ 明. The choice of barrier material strongly affects both $I_{c}$ and $R_{N}$ (typically poor conductors increase $R_{N}$ but reduce $I_{c}$ ) so it is appropriate to study in detail what properties

of the junction modify $I_{c}, R_{N}$, and $I_{c} R_{N}$. Indeed, it has been suggested 9 , that the maximum value of the product would be reached when the barrier material is close to a metal-insulator transition, so that the system is near the cross-over from an SNS to an SIS junction. In order to investigate such non-trivial barrier materials, we develop a microscopic model of a Josephson junction, which self-consistently incorporates the dynamical correlation effects of the electrons in calculating the conductance of the junction and the critical current.

Our model is also appropriate for ballistic junctions that have relatively pure barrier regions so that the extent of the barrier is smaller than its mean-free-path but larger than its proximity-effectinduced superconducting correlation length. Much work has progressed on these systems recently, with a concentration on Niobium-Indium Arsenide-Niobium junctions or Niobium-Silicon-Niobium junctions. Recent work includes an examination of subgap structures and current deficit $\$ 11$, quasiparticle reflection effects and spikes in the conductance 12 , effects of multiple Andreev reflection and noise in stacked junctions 1 , and an investigation of a tunable junction that can be altered from an ordinary junction to a $\pi$-junction by driving the barrier into a nonequilibrium state via a transverse electrical current14. The above-mentioned experimental and theoretical work has concentrated on nonequilibrium effects that occur at finite voltages. This contribution will address only equilibrium and linear-response properties of the microscopic model for Josephson junctions, but our work can be extended to examine such nonequilibrium effects as well. 
Josephson junctions consist of two superconducting regions coupled through an intermediate region which is not naturally superconducting. The main characteristics of a Josephson junction can be understood by just considering two superconductors coupled together with a single, energy independent transmissivity parameter. The dc Josephson effect, of a direct supercurrent through the junction at zero external voltage, and the ac Josephson effect of an oscillating, dissipative current at finite voltage were both predicted from such a simple modell . Further details of the I-V characteristics, such as excess current and sub-harmonic gap structure due to Andreev bound states were explained 5, e6, by matching boundary conditions across a step-function in the superconducting potential, and including an adjustable scattering potential at the interfaces 17,18 . Analytic calculations have been performed to provide current-phase relationships 192 , representing the barrier as a single scattering potential with no spatial extent. In this contribution we compare these traditional approaches with a more microscopic model.

Originally the term Josephson junction referred to superconductor - insulator - superconductor $(S I S)$ tunnel junctions, but here we use the term in its common usage to refer to all "weak links", including superconductor - normal metal - superconductor $(S N S)$ junctions. In the latter case, the barrier region can occupy tens to hundreds of nanometres, as the proximity effect ensures that the superconductivity extends into the barrier region, decaying on the scale of the coherence length, $\xi_{N}=\hbar v_{F} / k_{B} T$ when in the ballistic regime (where the coherence length is longer than the mean free path in the barrier).

In this paper, we present a method for studying the effects of the barrier region on the strength of superconductivity it can support, and hence on the supercurrent it can maintain. Effects of electron correlations are incorporated within the dynamic mean field theory 2326 , which leads to a local, frequency-dependent self-energy, which we allow to vary from one plane to the next, while assuming it to remain constant within individual planes. Hence the three-dimensional system becomes inhomogeneous in the one dimension where the current flows (which we label the z-direction). We model the system with two sets of $N_{S C}$ planes of superconducting material coupled each on one side to the bulk superconductor, and on the other side sandwiching $N_{b}$ planes of barrier material as depicted in Fig. 11. The total number of planes modeled self-consistently is $2 N_{S C}+N_{b}=N$. We note that while in this paper we concentrate on applying the method to Josephson junctions, our method would also be applicable to theoretical studies of correlated electrons at surfaces, single interfaces, or multiple interfaces, as first demonstrated by Potthoff and Noltinget.

An important advantage of our scheme is that the different (material specific) microscopic models 
that best describe any particular material can be coupled together across the planes. Hence a superconductor with electron-phonon coupling described by a Holstein modeles (or even using the appropriate $\alpha^{2} F$ in Migdal-Eliahberg theory) can be connected to a metallic region with impurities described by the Falicov-Kimball model (or a correlated Hubbard model), and so on. The local self energy for each plane is calculated independently, according to the model best-suited to the particular material. Once a set of local self-energies are evaluated for each plane, the Green's functions are calculated by finding the inverse of an infinite matrix which includes planes of bulk superconductor extending in the positive and negative z-direction. The inversion process, which is made tractable by a continued fraction representation, couples all of the different planes together, such that a change in the self energy on one plane affects the local interacting Green's functions on all other planes, particularly those nearby in real space.

In order for a supercurrent to flow, a phase gradient must be applied to the superconductor and across the barrier region. The critical current, $I_{c}$, is reached, when the planes with the lowest superconducting order, typically at the center of the barrier region, can no longer support the necessary phase gradient to maintain current continuity. In our model we find the supercurrent as a function of phase variation across the barrier, by solving the system self-consistently at each set of phases. The self-consistency is crucia 29.30, as the existence of a current flow affects the value of the superconducting order parameter, both inside and outside the barrier region.

Section II contains a detailed description of our method, including the physical approximations used, and the general computer algorithm. In Section III we analyze the current-phase relationships of our results in terms of the transmissivity of a barrier. The results presented in Section IV demonstrate the efficacy of the method to solve some simple models of Josephson junctions, using the Bogoliubov-de Gennes equations, where we demonstrate the effects of self-consistency, in particular on the superconducting order and electron density. Section V includes the results for barriers with impurity scattering, with a description of the calculations of normal-state resistance, and results of resistance and $I_{c} R_{N}$ products. We conclude in Section VI with some comments on the results, and suggestions of other situations well-suited to our model.

\section{METHOD}




\section{A. Model}

Our method consists of two stages. First, we determine the properties of the bulk boundary regions for a uniform system. When no current flows in the bulk (homogeneous) superconductor,

dynamical mean field theory 226 is employed to determine the local self energy, using the local approximation. When a uniform current is flowing, one must include a uniform phase variation in the superconducting order parameter, $\Delta(z)$, for the bulk system. Next, we solve the inhomogeneous problem self-consistently by iteration, for a number of planes, $N$, coupled on either side to the uniform bulk solution. That is, giving the plane an index, $\alpha$, and defining the central link of the junction to be between planes $\alpha=0$ and $\alpha=1$, we include $N_{b}$ barrier planes, surrounded by $N_{s c}$ self-consistently calculated superconductor planes on each side, such that $N=N_{b}+2 N_{s c}$ and planes with index, $\alpha<1-N / 2$ or $\alpha>N / 2$ are invariant homogeneous bulk planes (see Fig. 1). Typically, we solve systems with $N_{s c}=30$, which is significantly greater than the coherence length for our bulk superconductor ( $\xi=\hbar v_{F} / \Delta \approx 10 a$, where $a$ is the lattice spacing). We observe that, except when very close to $T_{c}$, the superconducting order has completely healed from its disruption at the interface, by the time we reach the planes at the bulk superconductor boundary (planes with $\alpha \approx \pm N / 2)$.

We describe the system with the following tight-binding model:

$$
\hat{H}=\sum_{i, j, \sigma} t_{i j} \hat{c}_{i \sigma}^{\dagger} \hat{c}_{j \sigma}+\sum_{i} U_{i}\left(\hat{c}_{i \uparrow}^{\dagger} \hat{c}_{i \uparrow}-\frac{1}{2}\right)\left(\hat{c}_{i \downarrow}^{\dagger} \hat{c}_{i \downarrow}-\frac{1}{2}\right)+\sum_{i, \sigma} U_{i}^{F K} \hat{c}_{i \sigma}^{\dagger} \hat{c}_{i \sigma} w_{i}
$$

where $\hat{c}_{i \sigma}^{\dagger}$ and $\hat{c}_{i \sigma}$ are fermionic operators which respectively create and destroy an electron of spin- $\sigma$ in a single Wannier (tight-binding) state on the lattice site $i$;

$$
t_{i j}=\left\{\begin{aligned}
\epsilon_{\alpha} & \text { if } i=j \text { on plane } \alpha, \\
-t_{\alpha} & \text { if } i \text { and } j \text { are neighboring sites on the same plane, } \alpha, \\
-\sqrt{t_{\alpha} t_{\alpha^{\prime}}} & \text { if } i \text { and } j \text { are neighboring sites on consecutive planes, } \alpha \text { and } \alpha^{\prime}, \\
0 & \text { otherwise, }
\end{aligned}\right.
$$

with $-t_{\alpha}$ the overlap or hopping integral for the $\alpha$-th plane, $\epsilon_{i}$ the local site energy, $U_{i}$ the renormalized on-site, Hubbard interaction energy, $U_{i}^{F K}$ the impurity potential, and $w_{i}=1$ if there is an impurity on site $i$, and $w_{i}=0$ otherwise. The magnitude of the hopping integral in the superconducting region, $t$, is constant, and defines our energy scale for the entire system $(t=1)$. In all results that we present here, the superconducting region has an attractive Hubbard interaction, 
$U_{i}=-2$ and no impurities $\left(w_{i}=0\right.$ for all sites on planes $\alpha<1-N_{b} / 2$ and $\left.\alpha>N_{b} / 2\right)$. We utilize the spinless Falicov-Kimball model to describe non-magnetic charge impurities within the barrier region. The interaction between dopant atoms and conduction electrons is attractive, and represented by a negative $U_{i}^{F K}$. The average impurity concentration within the barrier is given by, $\rho_{i m p}=\frac{1}{N_{b}}\left(\frac{a}{L}\right)^{2} \sum w_{i}$, where $N_{b}\left(\frac{L}{a}\right)^{2}$ is the total number of barrier sites. We also consider systems where there are no impurities within the barrier, but there is a Hubbard interaction for sites within the barrier, $U_{i}=U_{b}$, that differs from $U_{i}=-2$ in the superconductor. In addition, we have included systems where the hopping integral, $t_{\alpha}$, differs in the barrier region, and where the interfacial planes ( $\alpha=1-N_{b} / 2$ and $\alpha=N_{b} / 2$ only) have a non-zero local on-site potential, $\epsilon_{\alpha}=V_{\text {Int }}$.

\section{B. Green's Function Calculations}

We use the matrix formulation of Nambu 31 for the Green's function, $\underline{\underline{G}}\left(\mathbf{r}_{i}, \mathbf{r}_{j}, i \omega_{n}\right)$, between two lattice sites $\mathbf{r}_{i}$ and $\mathbf{r}_{j}$ at the Matsubara frequency, $i \omega_{n}=i \pi(2 n+1) k_{B} T$,

$$
\underline{\underline{G}}\left(\mathbf{r}_{i}, \mathbf{r}_{j}, i \omega_{n}\right)=\left(\begin{array}{cc}
G\left(\mathbf{r}_{i}, \mathbf{r}_{j}, i \omega_{n}\right) & F\left(\mathbf{r}_{i}, \mathbf{r}_{j}, i \omega_{n}\right) \\
\bar{F}\left(\mathbf{r}_{i}, \mathbf{r}_{j}, i \omega_{n}\right) & -G^{*}\left(\mathbf{r}_{i}, \mathbf{r}_{j}, i \omega_{n}\right)
\end{array}\right)
$$

and the corresponding local self energy,

$$
\underline{\underline{\Sigma}}\left(\mathbf{r}_{i}, i \omega_{n}\right)=\left(\begin{array}{cc}
\Sigma\left(\mathbf{r}_{i}, i \omega_{n}\right) & \phi\left(\mathbf{r}_{i}, i \omega_{n}\right) \\
\phi^{*}\left(\mathbf{r}_{i}, i \omega_{n}\right) & \left.-\Sigma^{*}\left(\mathbf{r}_{i}, i \omega_{n}\right)\right)
\end{array}\right) .
$$

The diagonal and off-diagonal Green's functions are defined respectively as:

$$
\begin{aligned}
& G\left(\mathbf{r}_{i}, \mathbf{r}_{j}, i \omega_{n}\right)=-\int_{0}^{\beta} d \tau \exp \left(i \omega_{n} \tau\right)\left\langle\mathrm{T}_{\tau} \hat{c}_{j \sigma}(\tau) \hat{c}_{i \sigma}^{\dagger}(0)\right\rangle \\
& F\left(\mathbf{r}_{i}, \mathbf{r}_{j}, i \omega_{n}\right)=-\int_{0}^{\beta} d \tau \exp \left(i \omega_{n} \tau\right)\left\langle\mathrm{T}_{\tau} \hat{c}_{j \uparrow}(\tau) \hat{c}_{i \downarrow}(0)\right\rangle
\end{aligned}
$$

where $\mathrm{T}_{\tau}$ denotes time-ordering in $\tau$ and $\beta=1 /\left(k_{B} T\right)$.

The self energies and Green's functions are coupled together through Dyson's equation,

$$
\underline{\underline{G}}\left(\mathbf{r}_{i}, \mathbf{r}_{j}, i \omega_{n}\right)=\underline{\underline{G}}^{(0)}\left(\mathbf{r}_{i}, \mathbf{r}_{j}, i \omega_{n}\right)+\sum_{l} \underline{\underline{G}}^{(0)}\left(\mathbf{r}_{i}, \mathbf{r}_{l}, i \omega_{n}\right) \underline{\underline{\Sigma}}\left(\mathbf{r}_{l}, i \omega_{n}\right) \underline{\underline{G}}\left(\mathbf{r}_{l}, \mathbf{r}_{j}, i \omega_{n}\right)
$$

where we have included the local approximation for the self energy, $\underline{\underline{\Sigma}}\left(\mathbf{r}_{i}, \mathbf{r}_{j}, i \omega_{n}\right)=\underline{\underline{\Sigma}}\left(\mathbf{r}_{i}, i \omega_{n}\right) \delta_{i j}$, [which can be relaxed if we use the Dynamical Cluster Approximation (DCA) 32]33]. The noninteracting Green's function, $\underline{\underline{G}}^{(0)}\left(\mathbf{r}_{i}, \mathbf{r}_{j}, i \omega_{n}\right)$ is diagonal in Nambu space, with upper diagonal component given by: 


$$
G^{(0)}\left(\mathbf{r}_{i}, \mathbf{r}_{j}, i \omega_{n}\right)=\int d^{3} \mathbf{k} \frac{\mathrm{e}^{i \mathbf{k} \cdot\left(\mathbf{r}_{i}-\mathbf{r}_{j}\right)}}{i \omega_{n}-\varepsilon_{\mathbf{k}}+\mu} .
$$

We emphasize that $\underline{\underline{G}}^{(0)}$ is the non-interacting Green's function and is not the effective medium of an equivalent atomic problem (see below for athe detailed algorithm used to solve the dynamical mean field theory). A major innovation in our work is to utilize an efficient hybrid real-space - momentumspace method for calculating the Green's functions from the set of local self-energies. We find this method to be much more powerful in solving systems with spatial variations or inhomogeneity, and it is also faster in bulk systems with current flow than a more conventional k-space integral technique.

Since the stacked planes have translational symmetry within the plane, the systems that we study are inhomogeneous in one direction only. We choose that direction to be labeled the z-axis, which is also the direction of current flow through the Josephson junction. The first stage of our method is to convert the problem from a three-dimensional system to a one-dimensional system following the algorithm of Potthoff and Nolting27. We perform a Fourier transform within the planes to determine the planar indexed Green's functions,

$$
\underline{\underline{G}}_{\alpha \beta}\left(i \omega_{n}, k_{x}, k_{y}\right)=\left(\frac{a}{L}\right)^{2} \sum_{x_{j}, y_{j}} \underline{\underline{G}}\left(\mathbf{r}_{i}, \mathbf{r}_{j}, i \omega_{n}\right) \exp \left[k_{x}\left(x_{j}-x_{i}\right)+k_{y}\left(y_{j}-y_{i}\right)\right],
$$

where $\alpha$ and $\beta$ denote distinct planes, defined by $\alpha=z_{i} / a, \beta=z_{j} / a$ and the summation is over all lattice sites, $\left(x_{j}, y_{j}\right)$, within the $\beta$-th plane. The self energy, $\underline{\underline{\Sigma}}_{\alpha}\left(i \omega_{n}\right)=\underline{\underline{\Sigma}}\left(z_{i}, i \omega_{n}\right)=\underline{\underline{\Sigma}}\left(\mathbf{r}_{i}, i \omega_{n}\right)$, is independent of the planar coordinates $x_{i}$ and $y_{i}$, so that Dyson's equation [Eq. (6)] becomes

$$
\underline{\underline{G}}_{\alpha \beta}\left(i \omega_{n}, k_{x}, k_{y}\right)=\underline{\underline{G}}_{\alpha \beta}^{(0)}\left(i \omega_{n}, k_{x}, k_{y}\right)+\sum_{\gamma} \underline{\underline{G}}_{\alpha \gamma}^{(0)}\left(i \omega_{n}, k_{x}, k_{y}\right) \underline{\underline{\Sigma}}_{\gamma}\left(i \omega_{n}\right) \underline{\underline{G}}_{\gamma \beta}\left(i \omega_{n}, k_{x}, k_{y}\right),
$$

with the summation over all planes, $\gamma$. The non-interacting planar Green's function, is similarly found by the Fourier transform

$$
\underline{\underline{G}}_{\alpha \beta}^{(0)}\left(i \omega_{n}, k_{x}, k_{y}\right)=\left(\frac{a}{L}\right)^{2} \sum_{x_{j}, y_{j}} \underline{\underline{G}}^{(0)}\left(\mathbf{r}_{i}, \mathbf{r}_{j}, i \omega_{n}\right) \exp \left[k_{x}\left(x_{j}-x_{i}\right)+k_{y}\left(y_{j}-y_{i}\right)\right] .
$$

The local Green's function, $\underline{\underline{G}}\left(\mathbf{r}_{i}, \mathbf{r}_{i}, i \omega_{n}\right)$, is required for calculating the self-consistent potentials, and the Green's function $\underline{\underline{G}}\left(\mathbf{r}_{i}, \mathbf{r}_{j}, i \omega_{n}\right)$ between two neighboring sites, $\mathbf{r}_{i}=\left(x_{i}, y_{i}, z_{i}\right)$ and $\mathbf{r}_{j}=$ $\left(x_{i}, y_{i}, z_{i} \pm a\right)$ is required for current calculations. These are given by the simple planar momentum integrals:

$$
\underline{\underline{G}}\left(\mathbf{r}_{i}, \mathbf{r}_{j}, i \omega_{n}\right)=\left(\frac{\pi}{a}\right)^{2} \int_{-\pi / a}^{\pi / a} \int_{-\pi / a}^{\pi / a} \underline{\underline{G}}_{\alpha, \beta}\left(i \omega_{n}, k_{x}, k_{y}\right) d k_{x} d k_{y}
$$


where again, $\alpha=z_{i} / a$ and $\beta=z_{j} / a$, and the phase factors in the integral have canceled as $x_{i}=x_{j}$ and $y_{i}=y_{j}$. Hence our goal is to find the interacting Green's functions, $\underline{\underline{G}}_{\alpha \beta}\left(i \omega_{n}, k_{x}, k_{y}\right)$.

We make a huge improvement (by one to two orders of magnitude) in the computational efficiency by transforming the two-dimensional planar momentum integral into a single integral over in-plane kinetic energy. In the case of nearest-neighbor hopping on a square lattice, the kinetic energy within the $\alpha$-th plane is given by

$$
\varepsilon_{\alpha}^{x y}=-2 t_{\alpha}\left[\cos \left(k_{x} a\right)+\cos \left(k_{y} a\right)\right]=\frac{t_{\alpha}}{t} \overline{\varepsilon^{x y}}
$$

where $t_{\alpha}$ is the hopping integral between two nearest-neighbor sites within the $\alpha$-th plane and $a$ is the lattice spacing. The effect of the in-plane kinetic energy is equivalent to an increase in the on-site energy, $\epsilon_{i}^{(0)} \mapsto \epsilon_{i}^{(0)}+\varepsilon_{\alpha}^{(x y)}$ which can vary between the different planes. The planar Green's functions only depend on the planar momentum via the normalized kinetic energy, $\overline{\varepsilon^{x y}}=$ $-2 t\left[\cos \left(k_{x} a\right)+\cos \left(k_{y} a\right)\right]$, such that $\underline{\underline{G}}_{\alpha \beta}\left(i \omega_{n}, k_{x}, k_{y}\right)=\underline{\underline{G}}_{\alpha \beta}\left(i \omega_{n}, \overline{\varepsilon^{x y}}\right)$. Hence, by using the twodimensional density of states, $\rho^{2 D}(\varepsilon)$, for a square lattice, the momentum integral is transformed into

$$
\left(\frac{a}{2 \pi}\right)^{2} \int_{-\pi / a}^{\pi / a} \int_{-\pi / a}^{\pi / a} G_{\alpha \beta}\left(i \omega_{n}, k_{x}, k_{y}\right) d k_{x} d k_{y}=\int_{-\infty}^{\infty} G_{\alpha \beta}\left(i \omega_{n}, \overline{\varepsilon^{x y}}\right) \rho^{2 D}\left(\overline{\varepsilon^{x y}}\right) d \overline{\varepsilon^{x y}}
$$

The specific hopping integral, $t_{\alpha}$, for each plane affects the on-site potential of a given plane in the continued fraction method [see Eq. (15) below], but does not contribute to the change of variables in the momentum integral.

Once the system is converted to a one-dimensional model, with nearest-neighbor hopping, the Green's functions can be solved rapidly by a continued fraction expansion, without recourse to another $k$-space integral for the $z$-direction. The continued fraction expansion is similar to the recursion method 34.35 , modified to include superconductivity 36 , but with three important differences. First, the method is much faster, as there is no need to expand about a site to obtain a new basis — the system is already one-dimensional in form. Second, there is no inaccuracy in the termination process, as the hopping integrals are given exactly in the model. The third point is an alteration, because the sites of interest are not at the end of a chain, but in the middle. This leads to a different set of continued fractions that must be calculated compared with the standard recursion method. In test runs, our method proves to be $4 \times 10^{5}$ times faster, and with machine precision accuracy, compared to a standard recursion method expansion which is terminated (due to memory limits) at an accuracy of one part in $10^{3}$ ! An alternate approach is to solve Dyson's matrix equation directly 
for a finite system, where the infinitely extended bulk boundaries can be mimicked by appropriate choice of potentials for the end planes. We have carried out such an approach as a comparison, but find it to be much slower (by a factor of 4000 for 60 planes than our method, and it grows like $N^{3}$ for large systems with $N$ planes) so we only describe the continued-fraction method below.

The equivalence of our method to the recursion method is that we calculate the Green's functions directly from a continued-fraction representation of the inverse of the Hamiltonian matrix in real space. That is, we find

$$
\underline{\underline{G}}_{\alpha \beta}\left(i \omega_{n}, \overline{\varepsilon^{x y}}\right)=\left(\begin{array}{ccccccc}
\ddots & \ddots & 0 & \vdots & \vdots & \vdots & \\
\ddots & i \omega_{n} \underline{\underline{1}}-\underline{\underline{a}}_{\alpha-2} & \underline{\underline{b}}_{\alpha-1} & 0 & 0 & 0 & \ldots \\
\cdots & \underline{\underline{b}}_{\alpha-1}^{\dagger} & i \omega_{n} \underline{\underline{1}}-\underline{\underline{a}}_{\alpha-1} & \underline{\underline{b}}_{\alpha} & 0 & 0 & \ldots \\
\cdots & 0 & \underline{\underline{b}}_{\alpha}^{\dagger} & i \omega_{n} \underline{\underline{1}}-\underline{\underline{a}}_{\alpha} & \underline{\underline{b}}_{n+1} & 0 & \ldots \\
\cdots & 0 & 0 & \underline{\underline{b}}_{\alpha+1}^{\dagger} & i \omega_{n} \underline{\underline{1}}-\underline{\underline{a}}_{\alpha+1} & \underline{\underline{b}}_{\alpha+2} & 0 \\
& \vdots & \vdots & 0 & \ddots & \ddots & \ddots
\end{array}\right)_{\alpha \beta}^{-1}
$$

where the matrices $\left\{\underline{\underline{a}}_{\alpha}\right\}$ are the total in-plane energies for a particular plane, given by

$$
\underline{\underline{a}}_{\alpha}=\left(\begin{array}{cc}
\epsilon_{\alpha}+\varepsilon_{\alpha}^{(x y)}+\Sigma_{\alpha}\left(i \omega_{n}\right)-\mu & \phi_{\alpha}\left(i \omega_{n}\right) \\
\phi_{\alpha}^{*}\left(i \omega_{n}\right) & -\epsilon_{\alpha}-\varepsilon_{\alpha}^{(x y)}-\Sigma_{\alpha}^{*}\left(i \omega_{n}\right)+\mu
\end{array}\right)
$$

and $\left\{\underline{\underline{b}}_{\alpha}\right\}$ couple the $\alpha-1$ th and $\alpha$ th planes,

$$
\underline{\underline{b}}_{\alpha}=\left(\begin{array}{cc}
-t_{\alpha-1, \alpha} & 0 \\
0 & t_{\alpha-1, \alpha}^{*}
\end{array}\right)
$$

The local planar Green's function, $\underline{\underline{G}}_{\alpha \alpha}\left(i \omega_{n}, \overline{\varepsilon^{x y}}\right)$, is readily evaluated as a combination of continued fractions (as in the renormalized perturbation expansion 37 ). We define the right-directed, $\underline{\underline{R}}_{\alpha}\left(i \omega_{n}\right)$, and left-directed $\underline{\underline{L}}_{\alpha}\left(i \omega_{n}\right)$ continued fractions from a plane, $\alpha$, recursively as

$$
\begin{aligned}
& \underline{\underline{R}}_{\alpha}\left(i \omega_{n}, \overline{\varepsilon^{x y}}\right)=i \omega_{n} \underline{\underline{1}}-\underline{\underline{a}}_{\alpha}-\underline{\underline{b}}_{\alpha+1} \underline{\underline{R}}_{\alpha+1}^{-1}\left(i \omega_{n}, \overline{\varepsilon^{x y}}\right) \underline{\underline{b}}_{\alpha+1}^{\dagger} \\
& \underline{\underline{L}}_{\alpha}\left(i \omega_{n}, \overline{\varepsilon^{x y}}\right)=i \omega_{n} \underline{\underline{1}}-\underline{\underline{a}}_{\alpha}-\underline{\underline{b}}_{\alpha}^{\dagger} \underline{\underline{L}}_{\alpha-1}^{-1}\left(i \omega_{n}, \overline{\varepsilon^{x y}}\right) \underline{\underline{b}}_{\alpha}
\end{aligned}
$$

The recursive calculation continues to infinity, but once it has been extended to planes in the uniform bulk medium, where $\alpha<1-N / 2$ or $\alpha>N / 2$, the coefficients at each level become constant. The effect of a constant phase gradient in $\phi$ is equivalent to a constant phase factor in the hopping integral, $t_{\alpha, \alpha+1}$, that does not change between planes in the bulk. Hence, by equating all 
$\underline{\underline{R}}_{\alpha}\left(i \omega_{n}, \overline{\varepsilon^{x y}}\right)$ as $\underline{\underline{R}}_{\infty}\left(i \omega_{n}, \overline{\varepsilon^{x y}}\right)$ for $\alpha>N / 2$ and $\underline{\underline{L}}_{\alpha}\left(i \omega_{n}, \overline{\varepsilon^{x y}}\right)$ as $\underline{\underline{L}}_{\infty}\left(i \omega_{n}, \overline{\varepsilon^{x y}}\right)$ for $\alpha<1-N / 2$ in the bulk limit, an exact terminator function can be calculated as the solution of a complex quadratic matrix equation:

$$
\begin{array}{r}
\underline{\underline{R}}_{\infty}\left(i \omega_{n}, \overline{\varepsilon^{x y}}\right) \underline{\underline{b}}_{\infty}^{\dagger-1} \underline{\underline{R}}_{\infty}\left(i \omega_{n}, \overline{\varepsilon^{x y}}\right)+\left[\underline{\underline{a}}_{\infty}-i \omega_{n} \underline{\underline{1}}\right] \underline{\underline{b}}_{\infty}^{\dagger-1} \underline{\underline{R}}_{\infty}\left(i \omega_{n}, \overline{\varepsilon^{x y}}\right)+\underline{\underline{b}}_{\infty}=\underline{\underline{0}} \\
\underline{\underline{L}}_{\infty}\left(i \omega_{n}, \overline{\varepsilon^{x y}}\right) \underline{\underline{b}}_{\infty}^{-1} \underline{\underline{L}}_{\infty}\left(i \omega_{n}, \overline{\varepsilon^{x y}}\right)+\left[\underline{\underline{a}}_{\infty}-i \omega_{n} \underline{\underline{1}}\right] \underline{\underline{b}}_{\infty}^{-1} \underline{\underline{L}}_{\infty}\left(i \omega_{n}, \overline{\varepsilon^{x y}}\right)+\underline{\underline{b}}_{\infty}^{\dagger}=\underline{\underline{0}}
\end{array}
$$

Note that the same terminator function is used for all sites in the intermediate layers, and the functions $\underline{\underline{R}}_{\alpha}$ and $\underline{\underline{L}}_{\alpha}$ calculated for one site are used in the calculation for the next site - so the number of computations required to find solutions for all sites can be less than $O(N)$.

There are two ways to solve this matrix quadratic equation. When we perform calculations on the real axis, without including a supercurrent, the matrix equation becomes analytically tractable to solve. On the imaginary axis, we find that it is numerically faster to simply find an iterative solution to these quadratic equations within the bulk medium. In most cases, accuracies of one part in $10^{10}$ can be achieved in ten iterations or less.

The continued fractions form the local planar Green's functions, according to

$$
\underline{\underline{G}}_{\alpha \alpha}\left(i \omega_{n}, \overline{\varepsilon^{x y}}\right)=\left\{i \omega_{n} \underline{\underline{1}}-\underline{\underline{a}}_{\alpha}\left(\overline{\varepsilon^{x y}}\right)-\underline{\underline{b}}_{\alpha}^{\dagger} \underline{\underline{L}}_{\alpha-1}^{-1}\left(i \omega_{n}, \overline{\varepsilon^{x y}}\right) \underline{\underline{b}}_{\alpha}-\underline{\underline{b}}_{\alpha+1} \underline{\underline{R}}_{\alpha+1}^{-1}\left(i \omega_{n}, \overline{\varepsilon^{x y}}\right) \underline{\underline{b}}_{\alpha+1}^{\dagger}\right\}^{-1}
$$

which, using Eqs.(17-18), can be simplified to

$$
\underline{\underline{G}}_{\alpha \alpha}\left(i \omega_{n}, \overline{\varepsilon^{x y}}\right)=\left[\underline{\underline{R}}_{\alpha}+\underline{\underline{L}}_{\alpha}-i \omega_{n} \underline{\underline{1}}+\underline{\underline{a}}_{\alpha}\right]^{-1}
$$

The Green's functions connecting neighboring planes, $\alpha$ and $\alpha \pm 1$, which are required to calculate the current flow, are given in two equivalent forms

$$
\begin{aligned}
& \underline{\underline{G}}_{\alpha, \alpha+1}\left(i \omega_{n}, \overline{\varepsilon^{x y}}\right)=-\underline{\underline{G}}_{\alpha \alpha}\left(i \omega_{n}, \overline{\varepsilon^{x y}}\right) \underline{\underline{b}}_{\alpha+1} \underline{\underline{R}}_{\alpha+1}^{-1}\left(i \omega_{n}, \overline{\varepsilon^{x y}}\right)=-\underline{\underline{L}}_{\alpha}^{-1}\left(i \omega_{n}, \overline{\varepsilon^{x y}}\right) \underline{\underline{b}}_{\alpha+1} \underline{\underline{G}}_{\alpha+1, \alpha+1}\left(i \omega_{n}, \overline{\varepsilon^{x y}}\right) \\
& \underline{\underline{G}}_{\alpha, \alpha-1}\left(i \omega_{n}, \overline{\varepsilon^{x y}}\right)=-\underline{\underline{G}}_{\alpha \alpha}\left(i \omega_{n}, \overline{\varepsilon^{x y}}\right) \underline{\underline{b}}_{\alpha}^{\dagger} \underline{\underline{L}}_{\alpha-1}^{-1}\left(i \omega_{n}, \overline{\varepsilon^{x y}}\right)=-\underline{\underline{R}}_{\alpha+1}^{-1}\left(i \omega_{n}, \overline{\varepsilon^{x y}}\right) \underline{\underline{b}}_{\alpha}^{\dagger} \underline{\underline{G}}_{\alpha-1, \alpha-1}\left(i \omega_{n}, \overline{\varepsilon^{x y}}\right)
\end{aligned}
$$

The local planar Green's functions enable us to calculate the self-energy and electron density, $n_{i}$, on each site in a given plane, $\alpha=z_{i} / a$, with the latter given by

$$
n_{i}=k_{B} T \sum_{\omega_{n}} \int_{-\infty}^{\infty} \rho^{2 D}\left(\overline{\varepsilon^{x y}}\right) \operatorname{Im}\left[G_{\alpha \alpha}\left(i \omega_{n}, \overline{\varepsilon^{x y}}\right)\right] d \overline{\varepsilon^{x y}}
$$

The current, $I_{\alpha, \alpha+1}$, which flows along each link between two neighboring planes, $\alpha$ and $\alpha+1$, in the $z$-direction is given by: 


$$
I_{\alpha, \alpha+1}=\frac{2 t e}{\hbar} k_{B} T \sum_{\omega_{n}} \int_{-\infty}^{\infty} \rho^{2 D}\left(\overline{\varepsilon^{x y}}\right) \operatorname{Im}\left[G_{\alpha, \alpha+1}\left(i \omega_{n}, \overline{\varepsilon^{x y}}\right)\right] d \overline{\varepsilon^{x y}} .
$$

A stringent convergence check for self-consistency, when there is a phase difference between the bulk superconductors, is that the current flow is constant from one plane to the next.

For the bulk boundary regions, the uniform variation of phase in the off-diagonal self energy, $\phi\left(\mathbf{r}_{i}\right)$ has the form, $\phi\left(\mathbf{r}_{i}\right)=\phi_{0} \exp \left[i \mathbf{q} \cdot \mathbf{r}_{i}\right]$, where the net superfluid momentum depends on $\mathbf{q}=\left(0,0, q_{z}\right)$ through $m v_{s}=\frac{\hbar}{a} \sin \left(q_{z} a\right)$ and leads to the following solution of Dyson's equation:

$$
G\left(\mathbf{r}_{i}, \mathbf{r}_{j}, i \omega_{n}\right)=\int d^{3} \mathbf{k} \frac{\left(i \omega_{n}+\varepsilon_{\mathbf{k}-\mathbf{q}}-\mu+\Sigma^{*}\left(i \omega_{n}\right)\right) \mathrm{e}^{i \mathbf{k} \cdot\left(\mathbf{r}_{i}-\mathbf{r}_{j}\right)}}{\left(i \omega_{n}-\varepsilon_{\mathbf{k}}+\mu-\Sigma\left(i \omega_{n}\right)\right)\left(i \omega_{n}+\varepsilon_{\mathbf{k}-\mathbf{q}}-\mu+\Sigma^{*}\left(i \omega_{n}\right)\right)-\left|\phi_{0}\left(i \omega_{n}\right)\right|^{2}}
$$

and

$$
F\left(\mathbf{r}_{i}, \mathbf{r}_{j}, i \omega_{n}\right)=\int d^{3} \mathbf{k} \frac{\phi_{0}\left(i \omega_{n}\right) \mathrm{e}^{i(\mathbf{k}-\mathbf{q}) \cdot\left(\mathbf{r}_{i}-\mathbf{r}_{j}\right)}}{\left(i \omega_{n}-\varepsilon_{\mathbf{k}}+\mu-\Sigma\left(i \omega_{n}\right)\right)\left(i \omega_{n}+\varepsilon_{\mathbf{k}-\mathbf{q}}-\mu+\Sigma^{*}\left(i \omega_{n}\right)\right)-\left|\phi_{0}\left(i \omega_{n}\right)\right|^{2}}
$$

where the diagonal self energy, $\Sigma\left(i \omega_{n}\right)$, is independent of site index, $\mathbf{r}_{i}$, in the bulk. We will need only the local, and nearest-neighbor bulk Green's functions in our calculations.

\section{Local self energy calculations}

In this contribution, the superconducting region is modeled by the negative- $\mathrm{U}$ Hubbard model within the Hartree-Fock (static mean-field) approximation. In this case, the local self energy is found from the local Green's functions by:

$$
\Sigma\left(\mathbf{r}_{i}, i \omega_{n}\right)=U T \sum_{\omega_{n}} G\left(\mathbf{r}_{i}, \mathbf{r}_{i}, i \omega_{n}\right)
$$

and

$$
\phi\left(\mathbf{r}_{i}, i \omega_{n}\right)=-U T \sum_{\omega_{n}} F\left(\mathbf{r}_{i}, \mathbf{r}_{i}, i \omega_{n}\right)
$$

where the instantaneous electron-electron interaction energy, $U$, leads to a time-independent self

energy. This procedure is identical to the conventional Bogoliubov-de Gennes approach 38 , which neglects retardation effects in the superconductor. As all sites within a plane are identical, the self energy need only be calculated once for each of the $N$ planes.

For planes within the barrier which include impurities, the dynamical mean field approximation says that the local (site) Green's function, $\underline{\underline{G}}\left(\mathbf{r}_{i}, \mathbf{r}_{i}, i \omega_{n}\right)$ is related to a local host Green's function 39 , $\underline{\underline{\mathcal{G}}}\left(\mathbf{r}_{i}, i \omega_{n}\right)$, via 


$$
\underline{\underline{\mathcal{G}}}\left(\mathbf{r}_{i}, i \omega_{n}\right)=\left[\underline{\underline{G}}^{-1}\left(\mathbf{r}_{i}, \mathbf{r}_{i}, i \omega_{n}\right)+\underline{\underline{\Sigma}}\left(\mathbf{r}_{i}, i \omega_{n}\right)\right]^{-1}
$$

The atomic Green's function, $\underline{\underline{G^{a t}}}\left(\mathbf{r}_{i}, i \omega_{n}\right)$, which will be equated to the local Green's function, $\underline{\underline{G}}\left(\mathbf{r}_{i}, \mathbf{r}_{i}, i \omega_{n}\right)$, in the dynamical mean field approximation, then satisfies

$$
\underline{\underline{G^{a t}}}\left(\mathbf{r}_{i}, i \omega_{n}\right)=\left(1-\rho_{i m p}\right) \underline{\underline{\mathcal{G}}}\left(\mathbf{r}_{i}, i \omega_{n}\right)+\rho_{i m p}\left[\underline{\underline{\mathcal{G}}}^{-1}\left(\mathbf{r}_{i}, i \omega_{n}\right)-U^{F K} \underline{\underline{1}}\right]^{-1}
$$

and the local self energy becomes

$$
\underline{\underline{\underline{\Sigma}}}\left(\mathbf{r}_{i}, i \omega_{n}\right)=\underline{\underline{\mathcal{G}}}^{-1}\left(\mathbf{r}_{i}, i \omega_{n}\right)-{\underline{\underline{G^{a t}}}}^{-1}\left(\mathbf{r}_{i}, i \omega_{n}\right)
$$

Starting from a local self energy, $\underline{\underline{\Sigma}}\left(\mathbf{r}_{i}, i \omega_{n}\right)$, and a local Green's function, $\underline{\underline{G}}\left(\mathbf{r}_{i}, \mathbf{r}_{i}, i \omega_{n}\right)$, Eqs. 31 .32, 33) can be employed to iteratively determine a new self energy $\underline{\underline{\Sigma}}\left(\mathbf{r}_{i}, i \omega_{n}\right)$ when the plane is described by the Falicov-Kimball model. The method, which is solved for a fixed concentration of impurities, $\rho_{i m p}$, is equivalent to the coherent potential approximation. The algorithm is summarized in Fig. 目.

\section{PHASE VARIATION}

Standard theory of Josephson junctions predicts the phase variation of the current in the weakcoupling limit to be $I(\theta)=I_{c} \sin (\theta)$ where $\theta$ is the phase difference across the barrier, and $I_{c}$ is the temperature-dependent critical current. Such a current variation arises from consideration of two superconductors with different phases being coupled by a single energy-independent transmission coefficient, corresponding to the tunneling of Cooper pairs across a barrier. A more general consideration 21 includes the Bogoliubov-de Gennes equations for a one-dimensional system of two superconductors coupled across a potential barrier, $V$. The strength of the barrier is measured by $Z=m V / \hbar^{2} k_{F}$ which determines the transmission coefficient, $\tau$, according to $\tau=1 /\left(1+Z^{2}\right)$. It

is found that Andreev bound states carry the current, whose variation with phase difference across the barrier leads to 21

$$
I=-G_{N} \frac{\pi \Delta_{0}}{2 e} \frac{\sin (\theta)}{\sqrt{1-\tau \sin ^{2}(\theta / 2)}} \tanh \left(\frac{\Delta_{0} \cos (\theta / 2)}{2 k_{B} T}\right) .
$$

This reproduces the weak-coupling result 19 in the limit of low transparency, $\tau \ll 1$, with

$$
I=-G_{N} \frac{\pi \Delta_{0}}{2 e} \sin (\theta) \tanh \left(\frac{\Delta_{0}}{2 k_{B} T}\right)
$$


and reproduces the formula for a point contact Josephson junction with perfect transparencyed, $\tau=1:$

$$
I=-G_{N} \frac{\pi \Delta_{0}}{e} \sin (\theta / 2) \tanh \left(\frac{\Delta_{0} \cos (\theta / 2)}{2 k_{B} T}\right)
$$

Interestingly, the maximum current is at a phase difference which approaches $\theta=\pi$ at low temperatures in the latter case, whereas traditional tunnel junctions have their maximum at $\theta=\pi / 2$.

In the more general case of a three-dimensional geometry22, the transmissivity depends on the angle of approach of the electron. The full current is obtained by an angular integral, $d \alpha$ over the Fermi surface. The complete calculation leads to:

$$
I(\theta)=-G_{N} \frac{\pi \Delta_{0}(T)}{2 e} \cdot \bar{R}_{N} \sin (\theta) \int_{-\pi / 2}^{\pi / 2} \frac{\sigma_{N}(\alpha) \cos (\alpha)}{\sqrt{1-\sigma_{N}(\alpha) \sin ^{2}(\theta / 2)}} \tanh \left(\frac{\Delta_{0}(T) \sqrt{1-\sigma_{N}(\alpha) \sin ^{2}(\theta / 2)}}{2 k_{B} T}\right) d \alpha
$$

where

$$
\bar{R}_{N}^{-1}=\int_{-\pi / 2}^{\pi / 2} \sigma_{N}(\alpha) \cos (\alpha) d \alpha
$$

The transmission probability for a single electron, which is proportional to its contribution to the conductivity, $\sigma_{N}$, (from a Landauer formula) depends on its angle of incidence, as well as the strength of scattering at the barrier.

The linear response of current due to a small phase difference, $I^{\prime}$, is given by:

$$
I^{\prime}=\left(\frac{\partial I}{\partial \theta}\right)_{\lim \theta \mapsto 0}=-G_{N} \frac{\pi \Delta_{0}(T)}{2 e} \tanh \left(\frac{\Delta_{0}(T)}{2 k_{B} T}\right)
$$

for both the one-dimensional and three-dimensional cases above. So if the barrier is parameterized by a single scattering potential, then the linear response supercurrent only depends on the normal state conductance according to Eq. (39) and the product of $I^{\prime} \cdot R_{N}$ is a constant, independent of the microscopic details of the barrier. More realistic treatments of the barrier region are expected to result in deviations from Eq. (39) and allow $I^{\prime} \cdot R_{N}$ to vary with the properties of the barrier.

Further differences arise between methods which treat the barrier as a single scattering potential and our self-consistent treatment of the order parameter and off-diagonal Green's functions within the barrier. Our results indicate that the effective scattering strength of the barrier, $Z$, changes with temperature and current flow. In general, the proximity effect, which enhances coupling between superconductors, is weakened as the current flow approaches the critical current. Hence at large 
current flow, the effective barrier is increased compared to its value in the linear response regime at close to zero current flow. The effect of self-consistency on the current-phase relationship appears to be more marked for weak barriers, where the current flow becomes relatively large.

We quantify the current response by making use of the linear response $I^{\prime}=(d I / d \theta)_{\lim \theta \mapsto 0}$, as well as the maximum current flow, $I_{c}$, through the barrier. In the weak-coupling limit $I_{c}$ is exactly equal to $I^{\prime}$, and our results show that in general the two are within $20 \%$ of each other, and scale almost identically with external parameters. As $I^{\prime}$ requires much less computational time to calculate, than $I_{c}$, we report values of $I^{\prime}$ for many of our results.

\section{BOGOLIUBOV-DE GENNES RESULTS}

To begin, we demonstrate how our model reproduces standard results, by using the Hartree-Fock approximation to calculate the self energy within the barrier region. As such, we are effectively self-consistently solving the full Bogoliubov-de Gennes equations for the system. In all the results that we present in this paper, the superconducting region is modeled with an attractive Hubbard interaction of $U=-2$ at half filling. The homogeneous bulk superconductor then has a critical temperature, $T_{c}=0.11$ and a zero temperature order parameter, $\Delta_{0}=0.198$.

\section{A. Barrier thickness}

We begin by solving for systems with a small attractive interaction within the barrier, $U_{b}=-0.5$, over a range of barrier thicknesses. This attraction is small enough that the bulk superconducting transition temperature of the barrier material is always less than any temperature we consider. Fig. 3 demonstrates the proximity effect, with the decay of the anomalous average at the center of the barrier as its width is increased. Fig. 1 is a log plot of the linear-response current and critical current against barrier thickness. As expected, both the linear-response current, $I^{\prime}$, and the critical current, $I_{c}$, drop rapidly when the number of planes within the barrier region is increased from 5 to 30 . The nearly constant slopes indicate that the decays are close to exponential. The exponent is approximately twice the bulk correlation length, given by $2 \xi \approx \hbar v_{f} / \Delta \approx 2 t a / \Delta \approx 10 a$, where $a$ is the lattice spacing.

Fig. 5 shows the normalized current as a function of phase difference across the barrier

region, for two different barrier thicknesses. The phase variation is compared to the sim- 
ple form $I(\theta) / I_{c}=\sin (\theta)$, which is appropriate in the weak-coupling limit, and $I(\theta) / I_{c}=$ $\left(I_{0} / I_{c}\right) \sin (\theta / 2) \tanh \left(\Delta \cos (\theta / 2) / 2 k_{B} T\right)$, the result for a point-contact junction, appropriate in the limit of high transmissivity. The curve for a thin junction falls outside these two limits, indicating that the effects of self-consistency and finite junction width are important in determining the current-phase relation for a Josephson junction. In particular, the calculated difference between a weakly coupled and strongly coupled junction lies in the opposite direction to the analytic formula on such a normalized curve. The actual magnitude of the critical current, is of course, greatly enhanced for the thinner barrier, as shown in Figs. 3 - 4 .

\section{B. Barrier interaction strength}

Fig. 6 demonstrates the proximity effect as a decay in the anomalous average, $F_{i i}=F\left(\mathbf{r}_{i}, \mathbf{r}_{i}, \tau=\right.$ $0^{+}$), within a barrier region of twenty planes. The proximity effect is seen to depend on the Hubbard interaction, $U_{b}$, for sites within the barrier. The corresponding critical currents, $I_{c}$ and linear response currents, $I^{\prime}$, are shown in Fig. 7. Note that in the cases where $U_{b}$ is negative, the barrier region is actually a superconductor in its normal state, above its transition temperature. In the case where $U_{b}=0$, the order parameter, $\Delta_{i}$, is exactly zero within the barrier. In the example where $U_{b}$ is positive, the order parameter actually switches sign within the barrier region, even when there is no external phase variation. The results of Fig. 7 indicate that such a switching in sign of the pairing potential, $\Delta_{i}$ has no marked effect on the transport properties, which depend on the continuously varying Green's functions of the system. Once again we find a systematic tracking of $I^{\prime}$ and $I_{c}$ with the strength of the Coulomb interaction, $U_{b}$, in the barrier.

\section{Barrier hopping integral}

Our method allows us to consider different hopping integrals in different regions (within planes or between planes). This would be appropriate when the barrier region has a density of states

at the Fermi surface, or a Fermi velocity that differs from that found in the normal state of the superconducting regions. When modeling such systems, the hopping integral between successive planes that have differing intraplanar hopping integrals, is taken as the geometric mean of the two planar values $\left(t_{\alpha, \alpha+1}=\sqrt{t_{\alpha} \cdot t_{\alpha+1}}\right)$.

Fig. 8 indicates that the critical current approximately scales with the hopping integral in the 
barrier region at low temperature. This effect dominates over any increased scattering at the interfaces due to Fermi velocity mismatch. In fact, when $t_{b}$ is large, the critical current is enhanced by a factor of $t_{b} / t$ over that found in the uniform bulk system $\left(t_{b}=1\right)$. The proximity effect results in a minimum of the anomalous average, $F_{i i}=F\left(\mathbf{r}_{i}, \mathbf{r}_{i}, \tau=0^{+}\right)=<c_{i \uparrow}\left(0^{+}\right) c_{i \downarrow}(0)>$, at the center of the barrier region. At low temperatures, Fig. 9 indicates that the system with a smaller hopping integral in the barrier, $t_{b}=1 / 2$, has a larger anomalous average than the uniform system (with $t_{b}=1$ ), due to its increase in density of states at the Fermi surface. As the temperature is increased, there is a crossover, with the system that has largest hopping integral in the barrier $\left(t_{b}=2\right)$, having the largest anomalous average just below the critical temperature, $T_{c}$. Such a crossover is due to the differing natural energy scales, $t_{b}$, in the barrier region. For the system with $t_{b}=1 / 2$, an actual temperature of $T=0.1$ corresponds to a temperature of 0.2 in the natural energy units of the barrier, $t_{b}$. When the temperatures are given in units of $t_{b}$, as shown in Fig. 10, a series of approximately parallel curves is seen, ordered according to the differing densities of states at the Fermi surface. The anomalous average, $F_{i i}$, plotted on the $y$-axis, does not require such scaling, as it is dimensionless.

\section{Local density of states}

It is interesting to observe the density of states, in particular the presence of states within the gap as shown in Fig. 11(a). The two major peaks correspond to Andreev bound states, which carry current, when there is a phase difference across the barrier region. The bound states can be seen to split in two, so that two inner peaks move towards each other, while two outer peaks move to the gap edge as the current flow increases. A careful examination of the curve corresponding to zero current reveals a great deal of structure, due to states trapped within the barrier region. The exact energies of the bound states depend on the width of the barrier, and the states arise in part from normal reflection of quasiparticles with non-zero momenta parallel to the planes $10,41,13$. The intraplanar momenta of the quasiparticles may lead to the states being unobservable as current peaks at the corresponding voltages in an I-V curve, where only quasiparticles travelling perpendicular to the planes are measured. In Fig. 12, we examine the bound states in more detail, for a system with ten barrier planes $\left(N_{b}=10\right)$. It is worthwhile noting that as the energy of the states within the gap approaches the gap edge, so they extend further away from the barrier, into the superconducting region [which begins after the fifth plane from the center $(\alpha>5)$ ]. The figure also demonstrates 
an alternating parity between states. The states with energy closest to zero have even parity, as demonstrated by a maximum amplitude at the central planes $(\alpha=0,1)$. The next higher energy bound states have a node at the central planes, so exhibit odd parity.

We can define a current-carrying local density of states such that the total current, $I_{\alpha, \alpha+1}$, between two successive planes, $\alpha$ and $\alpha+1$, is given by:

$$
I_{\alpha, \alpha+1}=\int i_{\alpha, \alpha+1}(E) d E .
$$

A plot of the function $i_{\alpha, \alpha+1}(E)$ between the two planes, $\alpha=0$ and $\alpha+1=1$, at the center of the barrier is shown in Fig. 13. It can be seen that the majority of the current is carried by the Andreev bound states, and that the two peaks that have separated from a single Andreev peak at zero phase difference, carry current in opposite directions. The states at positive energy carry equal and opposite current to the states at negative energy, but their occupation is much lower for low temperatures, $T<T_{c}$.

\section{E. Charge Depletion or Accumulation Regions}

By including an extra repulsive or attractive potential on the interfacial planes which connect the barrier to the superconductor, we are able to model in a very simple manner, some of the effects of a charge accumulation region or a Schottky Barrier. In our simple model there is a mirror symmetry for a half-filled band : a repulsive potential depletes the electron density in one layer, to reproduce some effects of a Schottky Barrier, while an attractive potential results in a charge accumulation region. The effect on the pairing potential and current density are the same, for equivalent potential strengths at half-filling. That is, if a repulsive interfacial potential, $V_{1}$, results in a reduction in electron density on a specific plane, then the attractive potential, $-V_{1}$, at the interface, causes an equal in magnitude increase of electron density at that plane, and results in a system with the same variation in order parameter, and equal current response.

Fig. 14(a) indicates the oscillations in the anomalous average resulting from a single layer barrier with $V_{0}= \pm 2 t$ and $V_{0}= \pm 4 t$. Notice that the proximity effect is reduced by the additional scattering potential, so the order parameter exhibits variation more like a step-function, with increasing barrier strength. We can think of the interfacial scattering as modifying the junction from SNS to SIS characteristics.

Fig. 15 depicts the effect on the electron density of a positive interfacial potential, which is like a Schottky barrier, since the electronic charge is depleted at the interface. The charge density also 
exhibits Friedel oscillations away from the barrier. The transformation $n_{i} \mapsto 2-n_{i}$ maps results for positive potential barrier to negative potential, $V_{0} \mapsto-V_{0}$, and will change the system to that of a charge accumulation region.

\section{CHARGE IMPURITY SCATTERING}

We model a barrier region with impurities by using the Falicov-Kimball model, as described in Section II, using Eq. (四) with $U^{F K}<0$ and using the self-consistency procedure of Eqs.(31. 33), as shown in Fig. 2. We carry out the calculations with an impurity concentration, $\rho_{i m p}$ that ranges from 0.01 to 0.2 . In the limit of $\rho_{i m p}=0$ there is no scattering in the barrier, and the results correspond to the Hubbard model with $U_{b}=0$. Impurities in the barrier region lead to an imaginary part for the frequency-dependent self energy and, since the lifetime of the quasiparticles at the Fermi surface becomes finite, to a non-Fermi liquid, characteristic of annealed disorder scattering.

\section{A. Current Responses}

The addition of a small number of impurities is seen in Fig. 16 to decrease the anomalous average in the barrier. The accompanying decrease in both critical current and linear response current, shown in Fig. 17 by the curves labeled $U_{F K}=-2$, is more severe. Addition of $10 \%$ impurities $\left(\rho_{i m p}=\right.$ 0.10) leads to a reduction in both current responses to approximately $1 / 3$ of their initial values, while the anomalous average remains at approximately $3 / 4$ its original amplitude. Anderson's theorem, which states that non-magnetic impurities do not detract from the superconducting properties of a system only holds for a spatially homogeneous system. A current flow breaks the symmetry, and a Josephson junction is inhomogeneous in one dimension, so the effects we observe do not violate Anderson's theorem. General considerations of Green's functions in a homogeneous system show that increasing the imaginary part of the electronic self-energy (hence reducing the quasi-particle lifetime) leads to a reduction in supercurrent for a given phase gradient. Hence it is expected that impurities would have a more deleterious effect on current responses than other superconducting properties, which is in the spirit of Anderson's theorem.

We look at the linear response current, $I^{\prime}$, as a function of temperature for a barrier with impurity scattering, in Fig. 18. The critical current remains slightly above zero until the bulk

critical temperature, $T_{c}$. As suggested by Eq. (39), we can extract an effective transmissivity, 
$\tau=G_{N} \cdot h / 2 e^{2}$ of the junction from $I^{\prime}$ using:

$$
G_{N}=I^{\prime} /\left\{\frac{\pi \Delta_{0}(T)}{2 e} \tanh \left(\frac{\Delta_{0}(T)}{2 k_{B} T}\right)\right\}
$$

where $\Delta_{0}(T)$ is the value of the order parameter on the last superconducting plane before the barrier region. Fig. 19 depicts the results, which demonstrate that a real barrier has lower transmissivity with increasing temperature. This can be understood from the variation of the self-consistent order parameter, which is reduced to zero within the barrier as temperature is increased. These results are impossible to predict using more conventional approaches, and only arise from a microscopic model that includes self-consistency.

\section{B. Resistance Calculations}

We find self-consistent solutions of a system in the normal state, with no current flow, by setting the order parameter to zero on all planes. The self energy of planes outside the barrier contains only a constant real part, as initially we carry out the calculations within the Hartree-Fock approximation. Given the set of local self energies, the Green's functions coupling any two planes are readily found, for any momentum parallel to the planes. We are interested in the longitudinal components in the z-direction (perpendicular to the uniform planes) of the conductivity matrix. We define the conductivity tensor for our effectively one-dimensional system, from the linear current response $I_{\alpha, \alpha+1}$ across a link between two planes, $\alpha$ and $\alpha+1$, due to an electric field, $E_{\beta, \beta+1}$ across all links between planes $\beta$ and $\beta+1$ :

$$
\sigma_{\alpha, \beta}=\frac{\partial I_{\alpha, \alpha+1}}{\partial E_{\beta, \beta+1}}
$$

We find the conductivity matrix with frequency component, $\nu$, neglecting vertex corrections (which is valid for homogeneous systems in the large dimensional limit) to be:

$$
\begin{aligned}
\sigma_{\alpha, \beta}(\nu)= & \frac{2}{\nu}\left(\frac{e a t}{\hbar}\right)^{2} \int_{-\infty}^{\infty} \rho^{2 D}\left(\varepsilon_{x y}\right) d \varepsilon_{x y} \int_{-\infty}^{\infty} \frac{d \omega}{2 \pi}\left\{\operatorname{Im}\left[G_{\alpha, \beta+1}\left(\omega, \varepsilon_{x y}\right)\right] \operatorname{Im}\left[G_{\beta, \alpha+1}\left(\omega+\nu, \varepsilon_{x y}\right)\right]+\right. \\
& \operatorname{Im}\left[G_{\alpha+1, \beta}\left(\omega, \varepsilon_{x y}\right)\right] \operatorname{Im}\left[G_{\beta+1, \alpha}\left(\omega+\nu, \varepsilon_{x y}\right)\right]-\operatorname{Im}\left[G_{\alpha, \beta}\left(\omega, \varepsilon_{x y}\right)\right] \operatorname{Im}\left[G_{\beta+1, \alpha+1}\left(\omega+\nu, \varepsilon_{x y}\right)\right]- \\
& \left.\operatorname{Im}\left[G_{\alpha+1, \beta+1}\left(\omega, \varepsilon_{x y}\right)\right] \operatorname{Im}\left[G_{\beta, \alpha}\left(\omega+\nu, \varepsilon_{x y}\right)\right]\right\}[f(\omega)-f(\omega+\nu)]
\end{aligned}
$$

where $f(\omega)$ is the Fermi-Dirac distribution function and $\rho^{2 D}\left(\varepsilon_{x y}\right)$ is the two-dimensional tightbinding density of states, used for the sum over momenta parallel to the planes. 
We are interested in the zero-frequency response, which is found from the appropriate limit of Eq. (43):

$$
\begin{aligned}
\sigma_{\alpha, \beta}(0)= & \frac{-1}{k_{B} T}\left(\frac{e a t}{\hbar}\right)^{2} \int_{-\infty}^{\infty} \rho^{2 D}\left(\varepsilon_{x y}\right) d \varepsilon_{x y} \int_{-\infty}^{\infty} \frac{d \omega}{2 \pi} \\
& \frac{\operatorname{Im}\left[G_{\alpha, \beta+1}\left(\omega, \varepsilon_{x y}\right)\right] \operatorname{Im}\left[G_{\beta, \alpha+1}\left(\omega, \varepsilon_{x y}\right)\right]-\operatorname{Im}\left[G_{\alpha, \beta}\left(\omega, \varepsilon_{x y}\right)\right] \operatorname{Im}\left[G_{\beta+1, \alpha+1}\left(\omega, \varepsilon_{x y}\right)\right]}{\cosh ^{2}\left(\omega /\left(2 k_{B} T\right)\right)}
\end{aligned}
$$

When calculating the resistance of the junction, it is important to be aware that for an inhomogeneous 1D system the current flow must be uniform but the electric field is not. The relationship

$$
I_{\alpha, \alpha+1}=I_{0}=\sum_{\beta} \sigma_{\alpha, \beta} E_{\beta, \beta+1}
$$

leads to

$$
E_{\beta, \beta+1}=\sum_{\alpha}\left(\sigma^{-1}\right)_{\beta, \alpha} I_{0}
$$

by multiplying on the left by the inverse of the conductivity tensor. The voltage across the junction is the sum of the electric fields across each link, multiplied by the lattice spacing, $a$, so we obtain the resistance,

$$
R_{N}=\frac{V}{I_{0}}=a \sum_{\alpha, \beta}\left(\sigma^{-1}\right)_{\beta, \alpha}
$$

given by the sum of components of the inverse conductivity tensor.

It is worthwhile pointing out, that where there is no imaginary part to the self energy, so there is no quasiparticle decay, the conductivity tensor consists of constant elements, $\sigma_{\alpha, \beta}=\sigma_{0}$. In such a region, the electric field required to produce a current flow approaches zero as the inverse of the system size, so the local conductivity becomes infinite. (That is, the sum of elements in one row of the conductivity tensor increases with system size.) However, the voltage drop across the region, given by the product of the electric field and length of perfect lead, remains constant (equal to $I_{0} a / \sigma_{0}$, so the resistance is non-zero 42 (equal to $a / \sigma_{0}$ ) while the local resistivity vanishes with large system size. In our calculations, where we neglect any lifetime effects of electrons outside the barrier region (in the Hartree-Fock approximation), there is still a contribution to the resistance of the junction from the 'perfect' leads, but the value of the contribution does not depend on the length of the leads, so it can be thought of as a contact resistance.

Fig. 20 indicates the variation of junction resistance with impurity concentration in the barrier. The resistance increases linearly with number of scattering sites for the small concentrations 
calculated, with the slope increasing with the strength of scatterers. The intercept is at a finite resistance, which corresponds to the resistance of an infinitely long, perfectly conducting lead, with conductivity tensor given by $\sigma_{\alpha, \beta}=\sigma_{0} \approx 1.25$. The resistance calculated for junctions with impurity scattering within the barrier region, does not change when the number of perfectly conducting planes on either side of the barrier is increased from one to twenty-five. Thereafter, numerical instabilities in the matrix inversion process make the calculations unreliable, but we can be confident that the answer already arrived at is the appropriate one for the infinite system.

The product $I_{c} \cdot R_{N}$ decreases with increasing concentration of impurities in the barrier for the examples shown. That is, the reduction in critical current is greater than the increase in resistance due to impurity scattering. A system which increases the $I_{c} \cdot R_{N}$ product is found by incorporating an extra (coherent) superconducting region within the barrier 3 . Such an $S N S^{\prime} N S$ or SIS IS structure has been examined, where an $S N S$ structure with 20 barrier planes has its central 6 barrier planes replaced with superconducting material, creating a barrier sandwich of 7 normal, 6 superconducting then 7 more normal planes. Both the critical current and linear response current increase by a factor of greater than two, while the normal state resistance is only reduced by $15 \%$ from its value for the SNS junction.

\section{CONCLUSIONS}

We have developed an effective method for calculating the equilibrium properties of Josephson junctions. We are able to examine the microscopic details of self-consistently solved systems through local and non-local Green's functions. The importance of self-consistency has previously been

shown29:30, so we go beyond standard 'potential barrier' models of junctions to include the effects of spatial correlation and local fluctuations on the self-consistent potentials.

Our results are interpreted in terms of a linear-response current, due to a small phase difference across the junction, as well as the critical current, being the maximum current that a junction can sustain. We find that a self-consistent microscopic determination of the potentials in the system for different phase differences results in current-phase behavior not predictable by standard fixed potential, semiclassical approaches. We study the effects of a number of properties of the barrier material, including its electron-electron interaction potential, its hopping integral and its charge impurity concentration. We also study interfacial scattering potentials, which mimic Schottky barriers and charge accumulation regions, and include barriers with a range of thicknesses. As well 
as quantifying the change in current response due to such modifications in the barrier, we also depict the alterations in the proximity effect for all cases, and charge variation in the case of an interfacial potential. In future work, we plan to model the charge redistribution at interfaces more realistically, by incorporating a non-local Coulomb potential, which will self-consistently determine an effective potential with the charge density for each plane. By such a model, we expect to discover if there is any significant charge redistribution as a junction passes through its superconducting transition, as suggested by Greene's group 4 .

We have also plotted the local density of states, to observe the bound states which occur at energies less than the bulk gap, within the barrier region. These states can be both current-carrying Andreev states, or states arising from normal reflections. Their evolution with position and phase variation is seen within our model, as is their contribution to the total current flow when a phase difference is applied. We plan in later work to show how the detailed structure of electronic states, apparent in these equilibrium results, will affect the I-V characteristics of a junction.

We have carried out resistance calculations for junctions with impurities in the barrier, and found that in general the reduction in critical current outweighs the increase in resistance due to charge impurities. We suggest that a junction of the form $S N S N S$, where a thin layer of superconductor is placed within an normal metal barrier, can increase the critical current of a junction dramatically, without markedly reducing its resistance. We are in a position to study how more subtle effects involving electronic correlation close to the metal-insulator can affect the $I_{c} \cdot R_{N}$ product. We plan to progress by making contact with specific materials, such as a Nb-InAs-Nb junction.

Our microscopic formulation will be particularly necessary when we proceed to study junctions created with high-temperature superconductor materials. The d-wave symmetry of the order parameter, with its directional dependence, results in behavior that is not addressed by models utilizing a single transmissivity function. While an appropriate non-local version of the Bogoliubov-de

Gennes equations can provide some insight into the properties of d-wave junctions29, 30, use of the $\mathrm{DCA} 32,33$ is necessary to tackle the problem in any realistic manner.

\section{ACKNOWLEDGEMENTS}

We are grateful to the Office of Naval Research for funding under grant number N00014-99-10328. 


\section{REFERENCES}

${ }^{1}$ B. D. Josephson, Phys. Lett. 1, 251 (1962).

${ }^{2}$ D. E. McCumber, J. App. Phys., 39, 2503 (1968).

${ }^{3}$ D. E. McCumber, J. App. Phys., 39, 3113 (1968).

${ }^{4}$ K. K. Likharev, Dynamics of Josephson Junctions and Circuits (Gordon and Breach Science Publishers, 1984).

${ }^{5}$ O. A. Mukhanov, V. K. Semenov, and K. K. Likharev, IEEE Trans. Magn. MAG-23, 759 (1987).

${ }^{6}$ K. K. Likharev, in Applications of Superconductivity, ed. by H. Weinstock (Kluwer, Dordrecht, 1999).

${ }^{7}$ V. Patel and J. E. Lukens, IEEE Trans. on Appl. Supercond. 9, 3247 (1999).

${ }^{8}$ M. Yu. Kupriyanov, and K. K. Likharev, Sov. Phys. Usp. 33, 340 (1990).

${ }^{9}$ S. J. Berkowitz, Y. M. Zhang, W. H. Mallison, K. Char, E. Terzioglu, and M. R. Beasley, Appl. Phy. Lett. 69, 3257 (1996).

${ }^{10}$ A. S. Barrera and M. R. Beasley, IEEE Trans. Magn. MAG-23, 866 (1987).

${ }^{11}$ A. Chrestin, T. Matsuyama, and U. Merkt, Phys. Rev. B 55, 8457 (1997).

${ }^{12}$ G. Bastian, E. O. Göbel, A. B. Zorin, H. Schulze, J. Niemeyer, T. Wiemann, M. R. Bennet, and K. E. Singer, Phys. Rev. Lett. 81, 1686 (1998).

${ }^{13}$ M. Thomas, H.-R. Blank, K. C. Wong, H. Kroemer, and E. Hu, Phys. Rev. B 58, 11676 (1998); A. Jacobs, R. Kümmel, and H. Plehn, Superlattices and Microstructures 25, 669 (1999).

14 J. J. A. Baselmans, A. F. Morpurgo, B. J. van Wees, and T. M. Klapwijk, Nature, 397, 43 (1999); F. K. Wilhelm, G. Schön, and A. D. Zaikin, Phys. Rev. Lett. 81, 1682 (1998); J. J. A. Baselmans, A. F. Morpurgo, B. J. van Wees, and T. M. Klapwijk, Superlattices and Microstructures, 25, 973 (1999).

${ }^{15}$ G. E. Blonder, M. Tinkham and T. M. Klapwijk, Phys. Rev. B 25, 4515 (1982).

${ }^{16}$ T. M. Klapwijk, G. E. Blonder and M. Tinkham, Physica 109-110B, 1657 (1982). 
${ }^{17}$ G. B. Arnold, Phys. Rev. B 17, 3576 (1978).

${ }^{18}$ E. L. Wolf and G. B. Arnold, Phys. Reports 91, 31 (1982).

${ }^{19}$ V. Ambegaokar and A. Baratoff, Phys. Rev. Lett. 10, 486 (1963).

${ }^{20}$ I. O. Kulik and A. N. Ome'lyanchuk, Fiz. Nizk. Temp. 4, 296 (1978) [Sov. J. Low Temp. Phys. 4, 142 (1978)].

${ }^{21}$ A. Furusaki, Superlattices and Microstructures (UK) 25, 809 (1999).

${ }^{22}$ Y. Tanaka and S. Kashiwaya, Phys. Rev. B 56, 892 (1997).

${ }^{23}$ W. Metzner and D. Vollhardt, Phys. Rev. Lett. 62, 324 (1989)

${ }^{24}$ M. Jarrell, Phys. Rev. Lett. 69, 168 (1992).

${ }^{25}$ J. K. Freericks, M. Jarrell, and D. J. Scalapino, Europhys. Lett. 25, 37 (1994).

${ }^{26}$ J. K. Freericks, Phys. Rev. B 50, 403 (1995).

${ }^{27}$ M. Potthoff and W. Nolting, Phys. Rev. B 59, 2549 (1999).

28 T. Holstein, Ann. Phys. 8, 352 (1959).

${ }^{29}$ A. M. Martin and J. F. Annett, Phys. Rev. B 57, 8709 (1998).

${ }^{30}$ J. J. Hogan-O'Neill, A. M. Martin and J. F. Annett, Phys. Rev. B 60, 3568 (1999).

${ }^{31}$ Y. Nambu, Phys. Rev. 117, 648 (1960).

${ }^{32}$ M. H. Hettler, A. N. Tahvildar-Zadeh, M. Jarrell, Th. Prushke, and H. R. Krishnamurthy, Phys. Rev. B 58, R7475 (1998).

33 Th. Maier, M. Jarrell, Th. Prushke, and J. Keller, preprint cond-mat/9906253 (1999).

${ }^{34}$ R. Haydock in Solid State Physics, Vol. 35 (Academic Press, 1980).

35 The Recursion Method and Its Applications Springer Series in Solid State Sciences 58 (Springer - Verlag, 1984).

${ }^{36}$ G. Litak, P. Miller and B. L. Györffy, Physica C, 251, 263 (1995).

${ }^{37}$ E. N. Economou, Green's Functions in Quantum Physics, App.B (Springer-Verlag, Berlin, 1983). 
${ }^{38}$ P. G. de Gennes, Superconductivity of Metals and Alloys (Addison-Wesley, 1966).

${ }^{39}$ U. Brandt and C. Mielsch, Z. Phys. B 75, 365 (1989).

${ }^{40}$ O. S̆ipr and B. L. Györffy, J. Phys. C 8, 169 (1996).

${ }^{41}$ R. Kümmel, H. Plehn, and U. Schüssler, Proceedings of 23rd International Conference on the Physics of Semiconductors 4, 3387 (1996).

${ }^{42}$ X. -G. Zhang and W. H. Butler, Phys. Rev. B, 55, 10308 (1997).

${ }^{43}$ A. Brinkman and A. A. Golubov, cond-mat/9912109 (unpublished).

${ }^{44}$ L. H. Greene, J. F. Dorsten, I. V. Roschchin, A. C. Abeyta, T. A. Tanzer, G. Kuchler, W. L. Feldmann, and P. W. Bohn, Czech. Journ. of Phys. 46, 3115 (1996), and unpublished. 


\section{FIGURES}

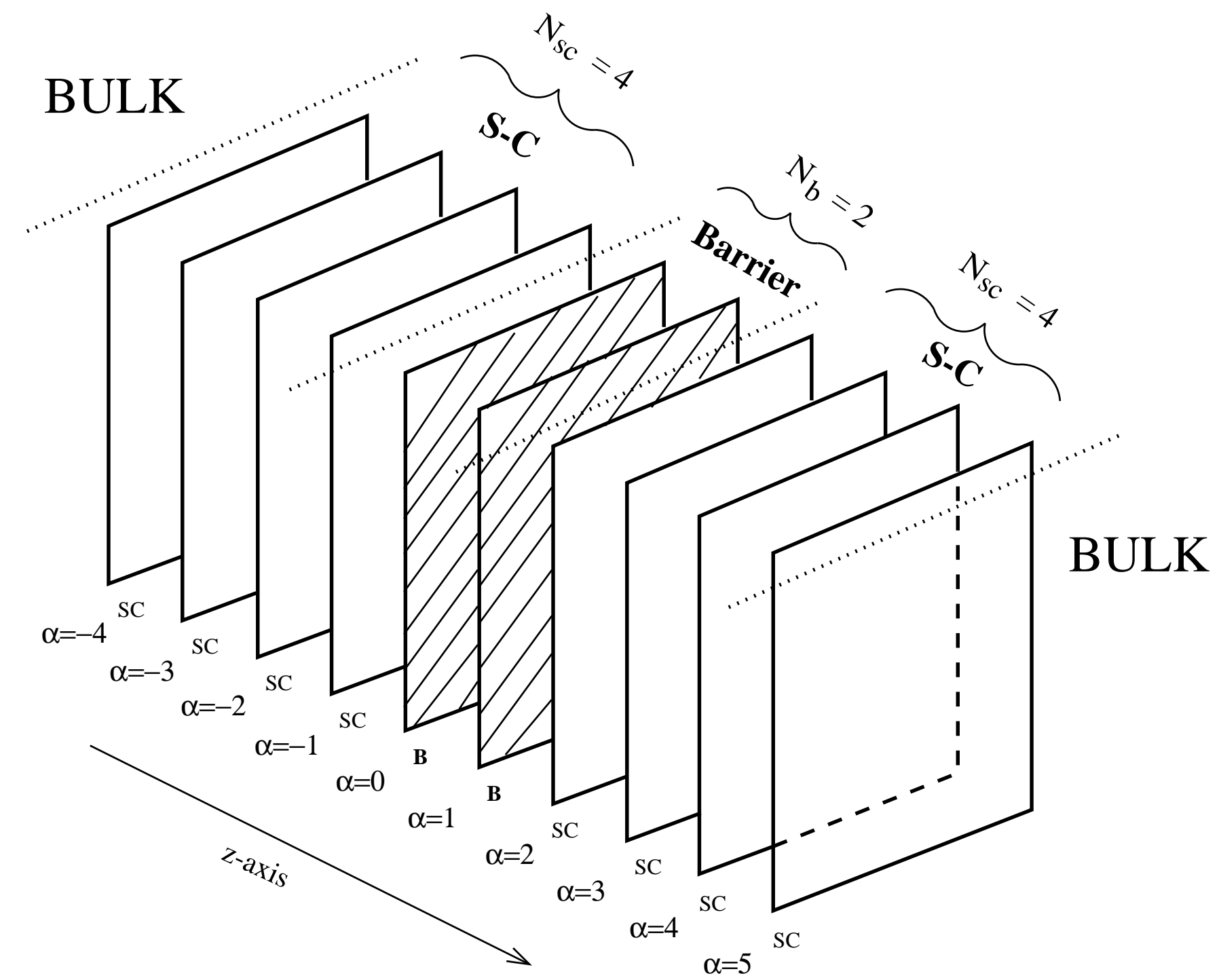

FIG. 1. Microscopic stacked planar geometry of a Josephson junction. The sandwich of $N=10$ planes; $N_{s c}=4$ superconducting planes coupled to a bulk superconductor on the left and $N_{b}=2$ barrier planes on the right, followed by a further $N_{s c}=4$ superconducting planes coupled to another bulk superconductor on the right. The system is allowed to have spatial inhomogeneity only within the $N$ modeled planes, but the calculations are always for an infinite system. 
Equate atomic and

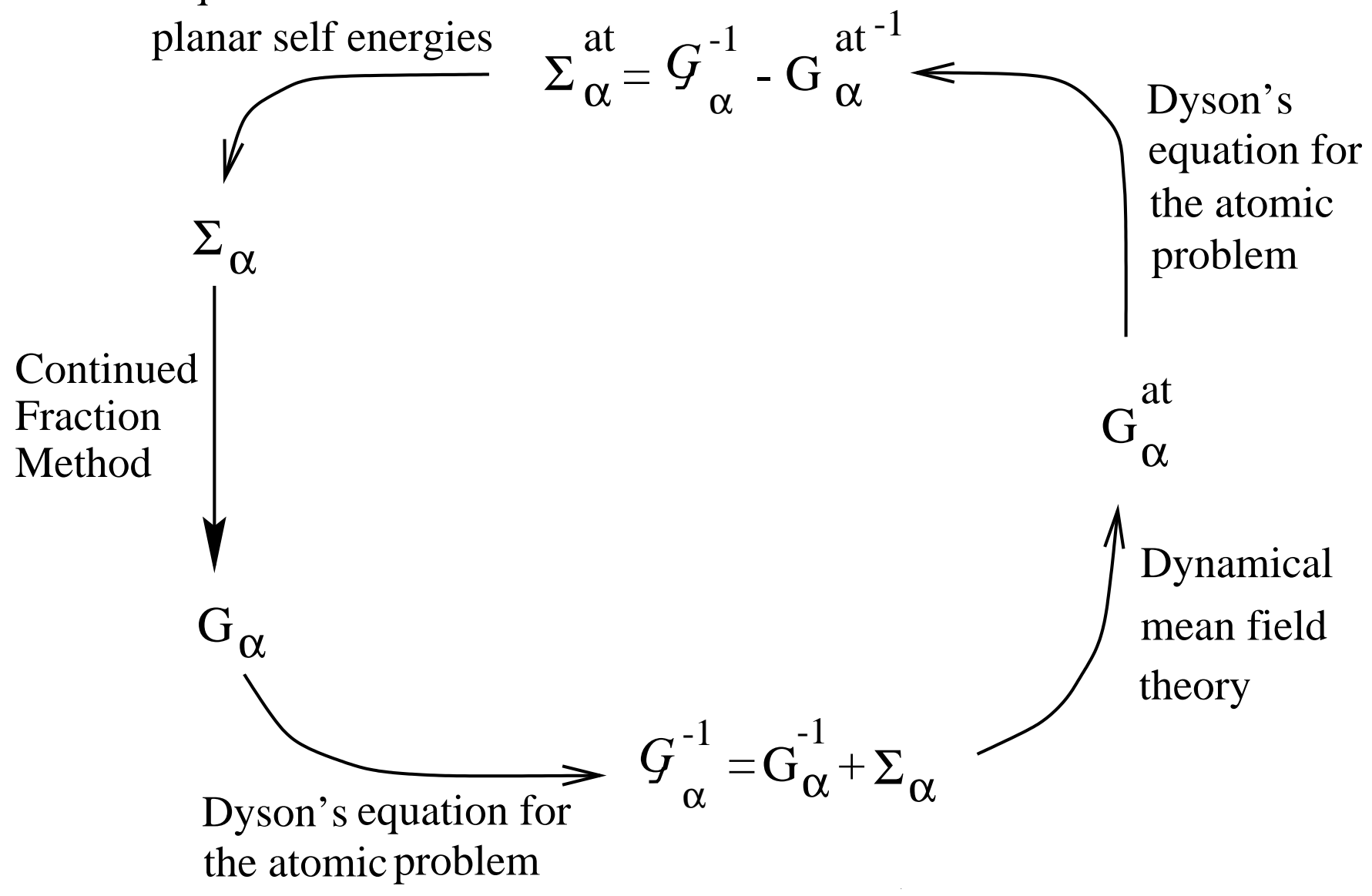

FIG. 2. Diagram of the iteration procedure, where the dynamical mean field theory is used to calculate local self-energies from local Green's functions. In the case of the Hartree-Fock approximation for the self energy, the dynamical mean field theory step is trivial, [see Eqs. (29 30)]. 


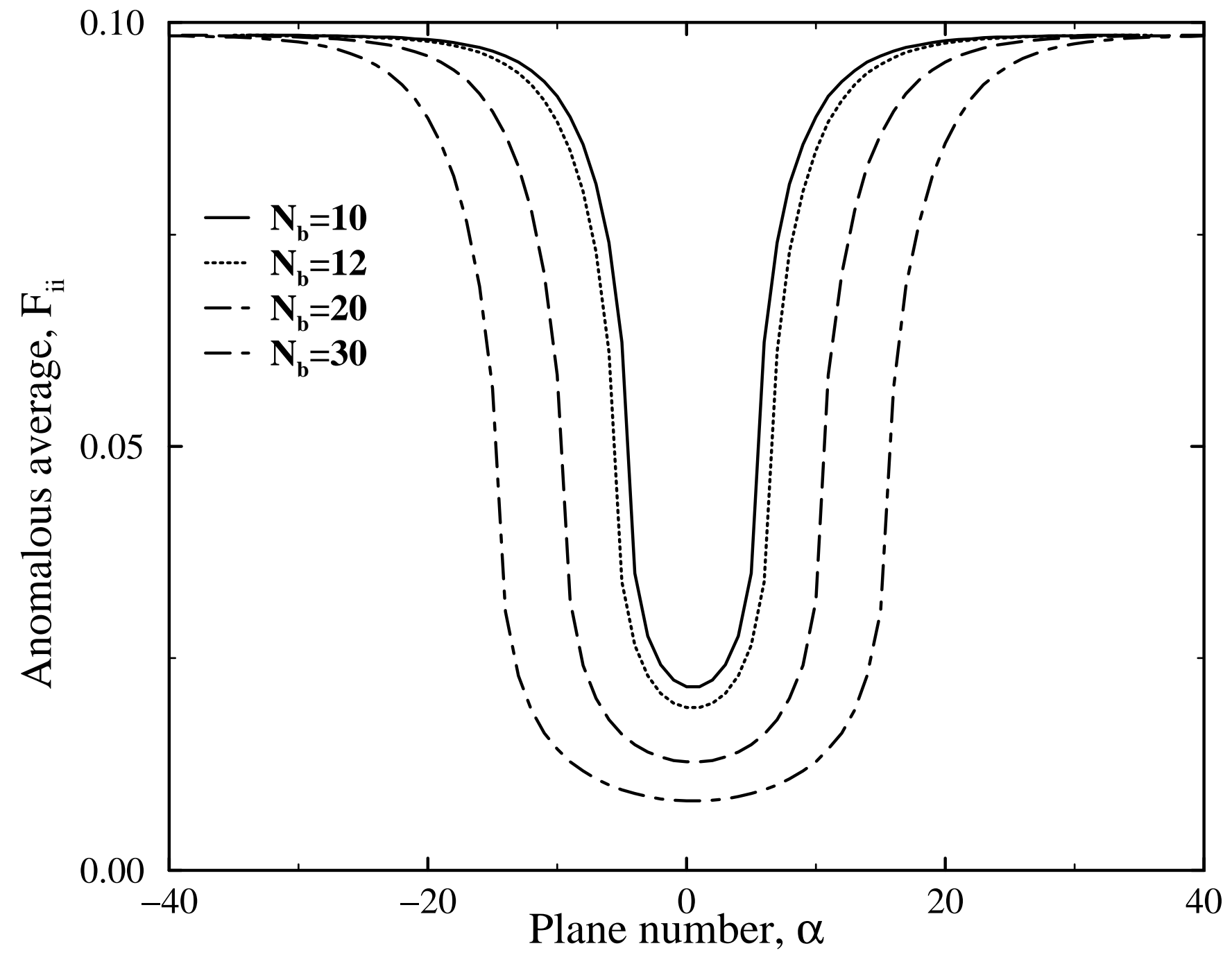

FIG. 3. Plot of the decay of the anomalous average due to the proximity effect in the barrier region. Note that the order parameter, $\Delta$ is equal to $2 F_{i i}=2 F\left(\mathbf{r}_{i}, \mathbf{r}_{i}, \tau=0^{+}\right)$in the superconducting region, where $U=-2$, and is equal to $F_{i i} / 2$ in the barrier region, as $U_{b}=-1 / 2$. It is the anomalous Green's function, $F_{i i}$, rather than the order parameter, $\Delta_{i}$, that is continuous throughout the system. 


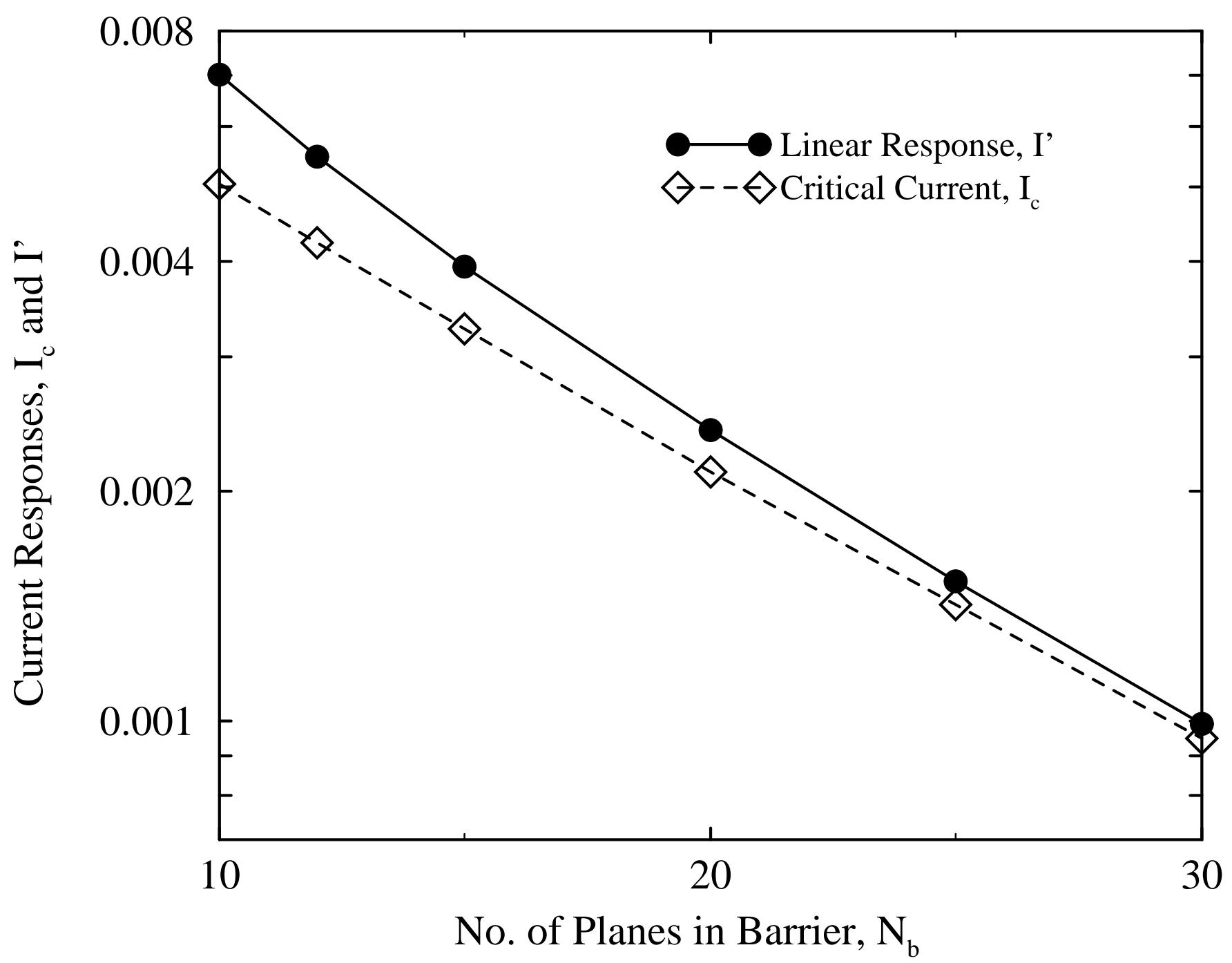

FIG. 4. Exponential decay of the linear response current, $I^{\prime}$, and critical current, $I_{c}$ with increasing barrier thickness. Note how both $I^{\prime}$ and $I_{c}$ systematically track with each other, and agree to within $20 \%$ for cases considered here. 


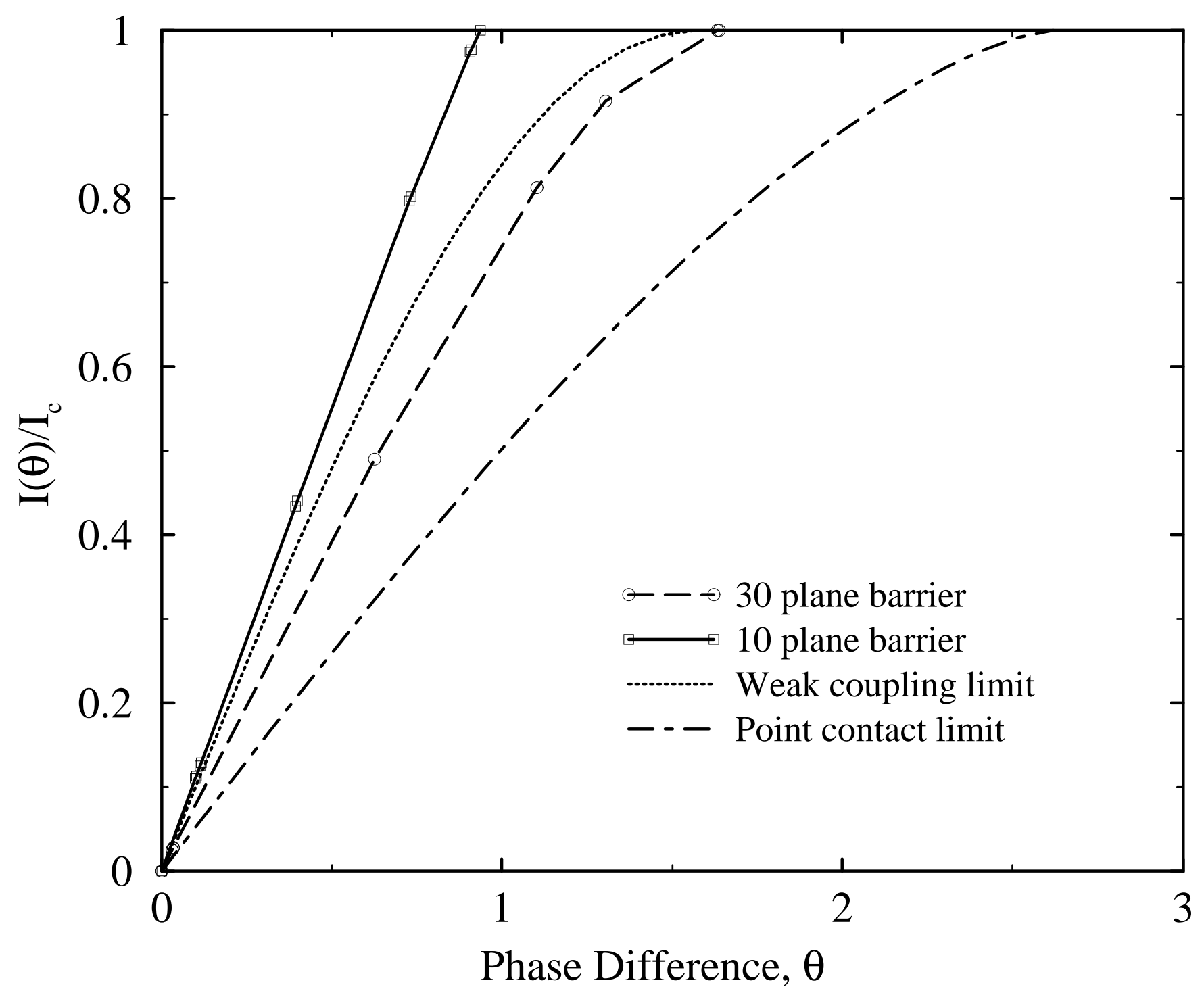

FIG. 5. Current flow as a function of phase difference across the barrier for a thin and a thick junction, compared to analytic results for a low transmissivity (dotted line) and a high transmissivity (chain-dashed line) junction. Notice how the results for a thin junction lie outside of the results for the two analytic limiting cases. This shows how including self-consistency and a microscopic model of the barrier width can affect the current-phase relation. 


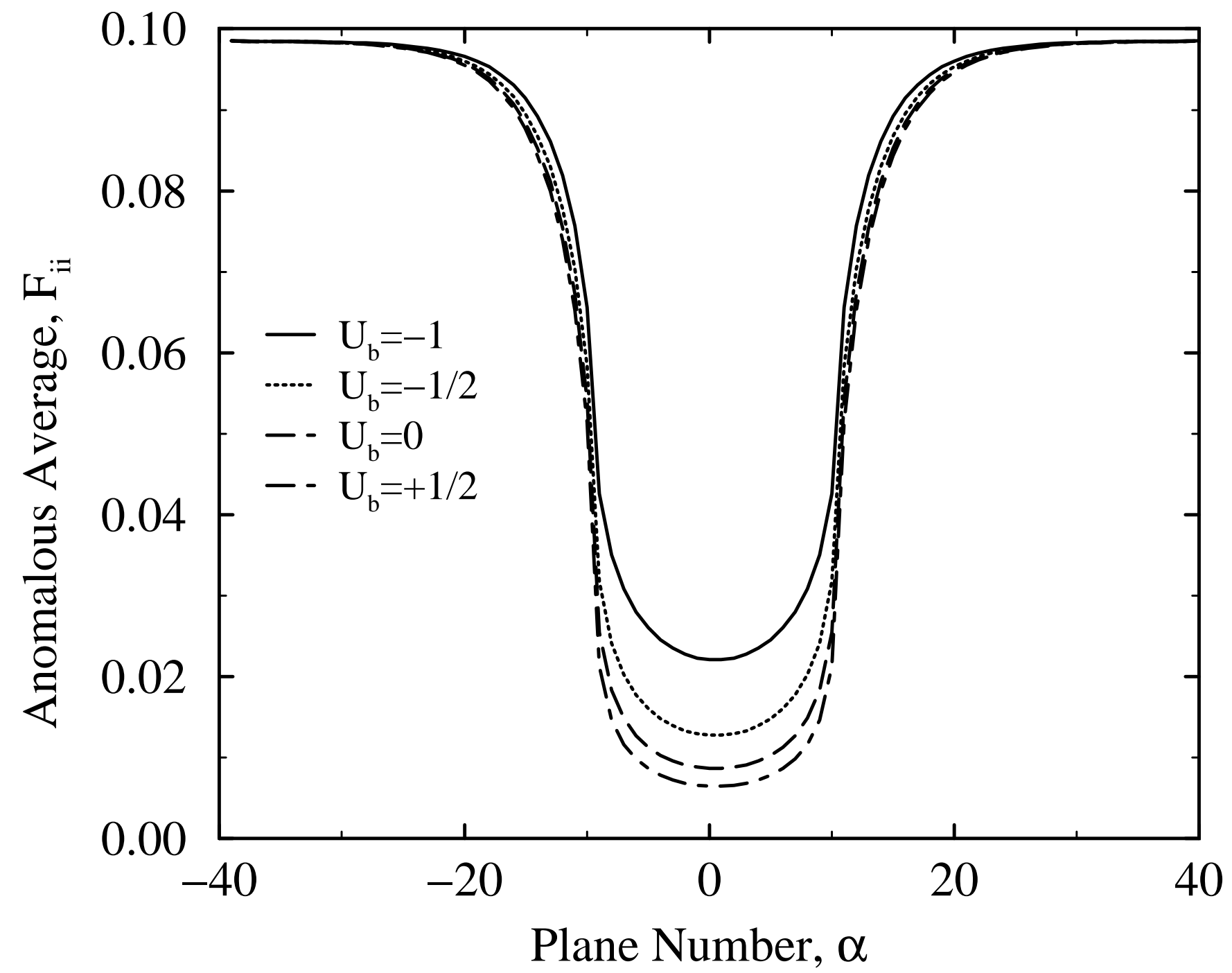

FIG. 6. The decay of the anomalous average within the barrier region as a function of the Hubbard interaction, $U_{b}$. Note how the anomalous average decays more rapidly as the Coulomb interaction in the barrier, $U_{b}$, increases in value (and becomes repulsive). 


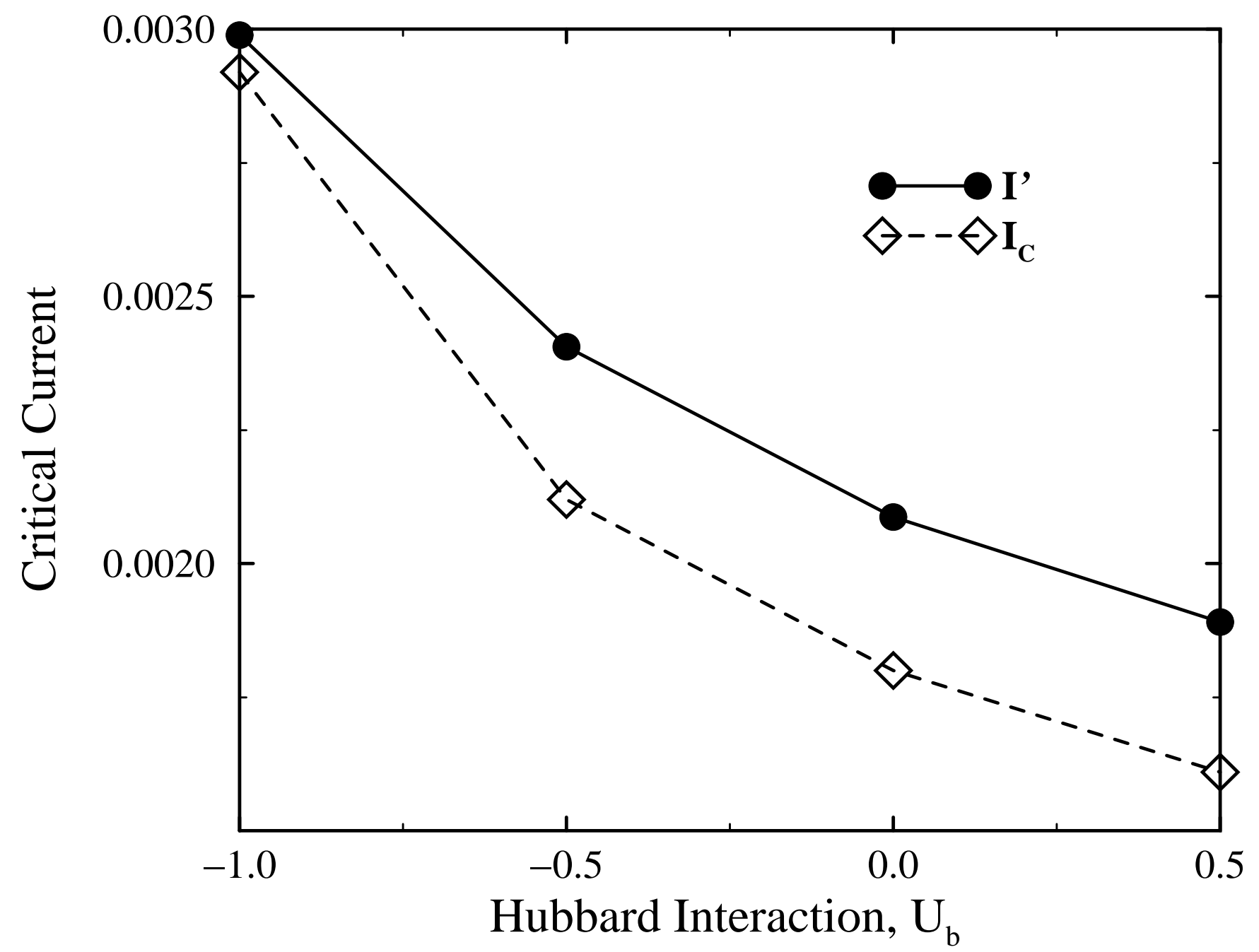

FIG. 7. Linear response current, $I^{\prime}$, and critical current, $I_{c}$, as a function of the Coulomb interaction in the barrier, $U_{b}$. Note how both critical currents fall with $U_{b}$, and note that there is no apparent discontinuity through $U_{b}=0$, where the sign of $\Delta$ changes within the barrier. 


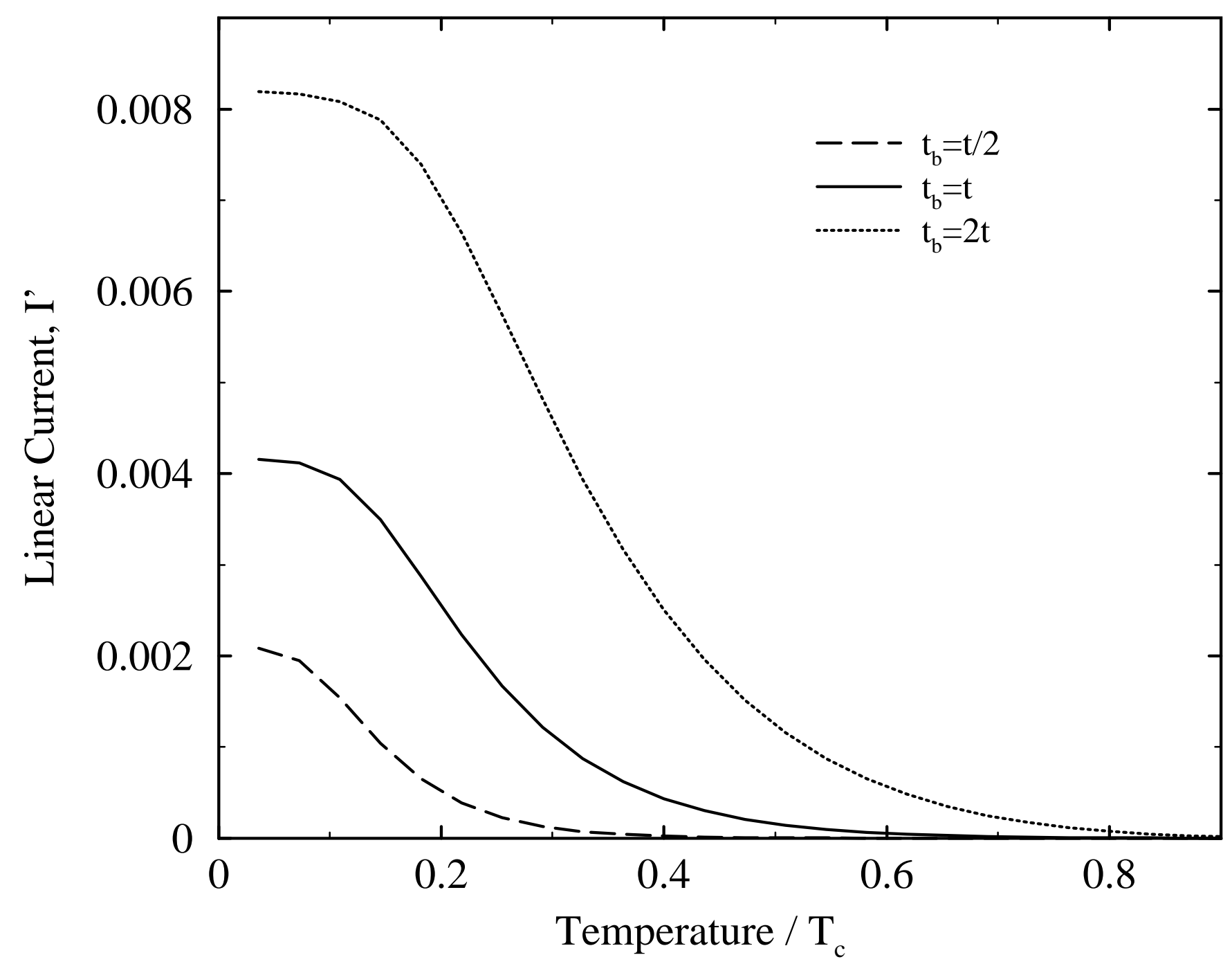

FIG. 8. Linear current, $I^{\prime}$, versus temperature for barriers with a hopping integral, $t_{b}$, that can differ from the hopping integral, $t$, between planes of the superconductor. Note how $I^{\prime}$ scales with $t_{b}$ in the low-temperature regime. 


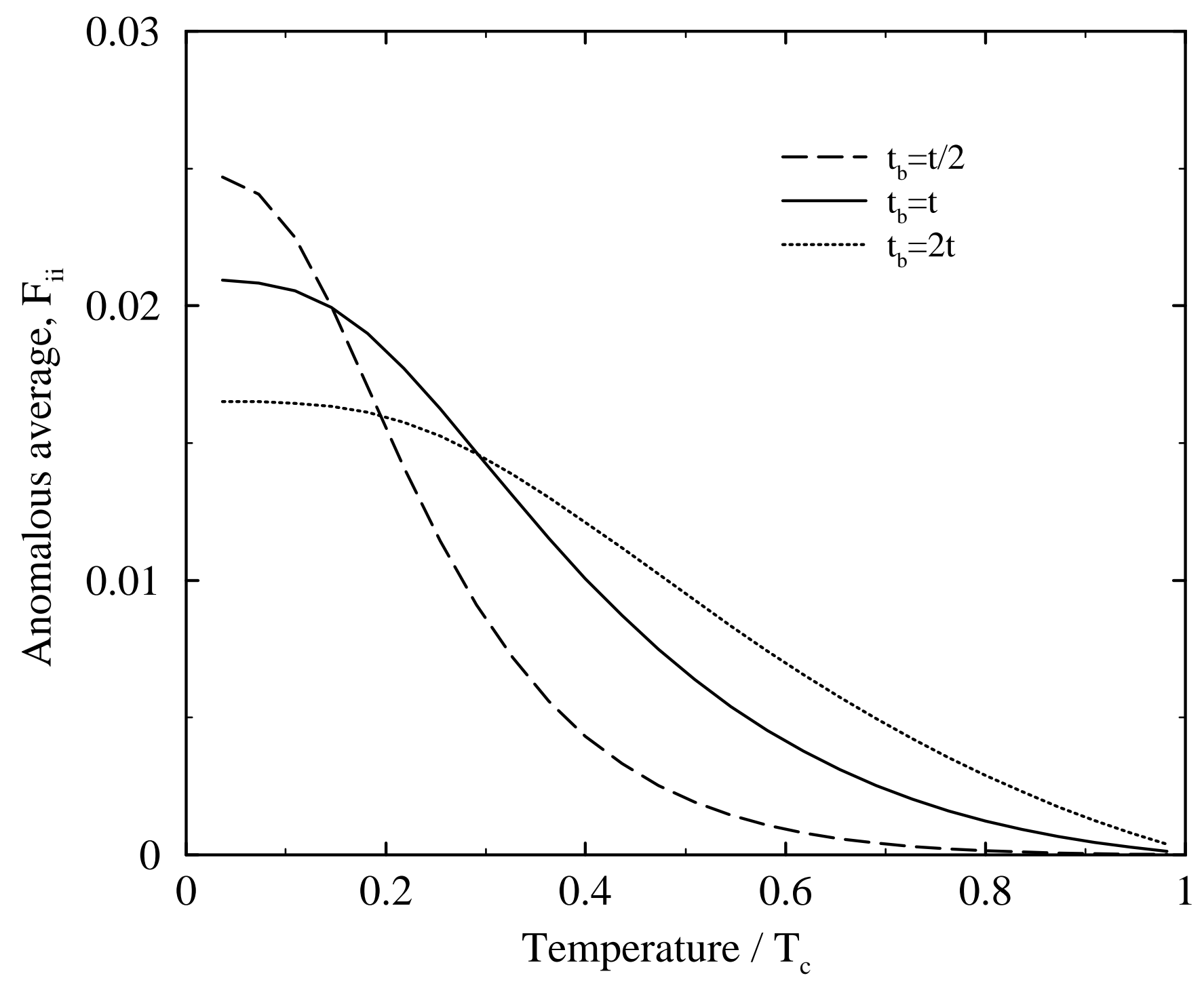

FIG. 9. The variation with temperature of the anomalous average, $F_{i i}=F\left(\mathbf{r}_{i}, \mathbf{r}_{i}, \tau=0^{+}\right)$, for the plane at the center of the barrier region. The different curves are results for different values of the barrier hopping integral, $t_{b}$. 


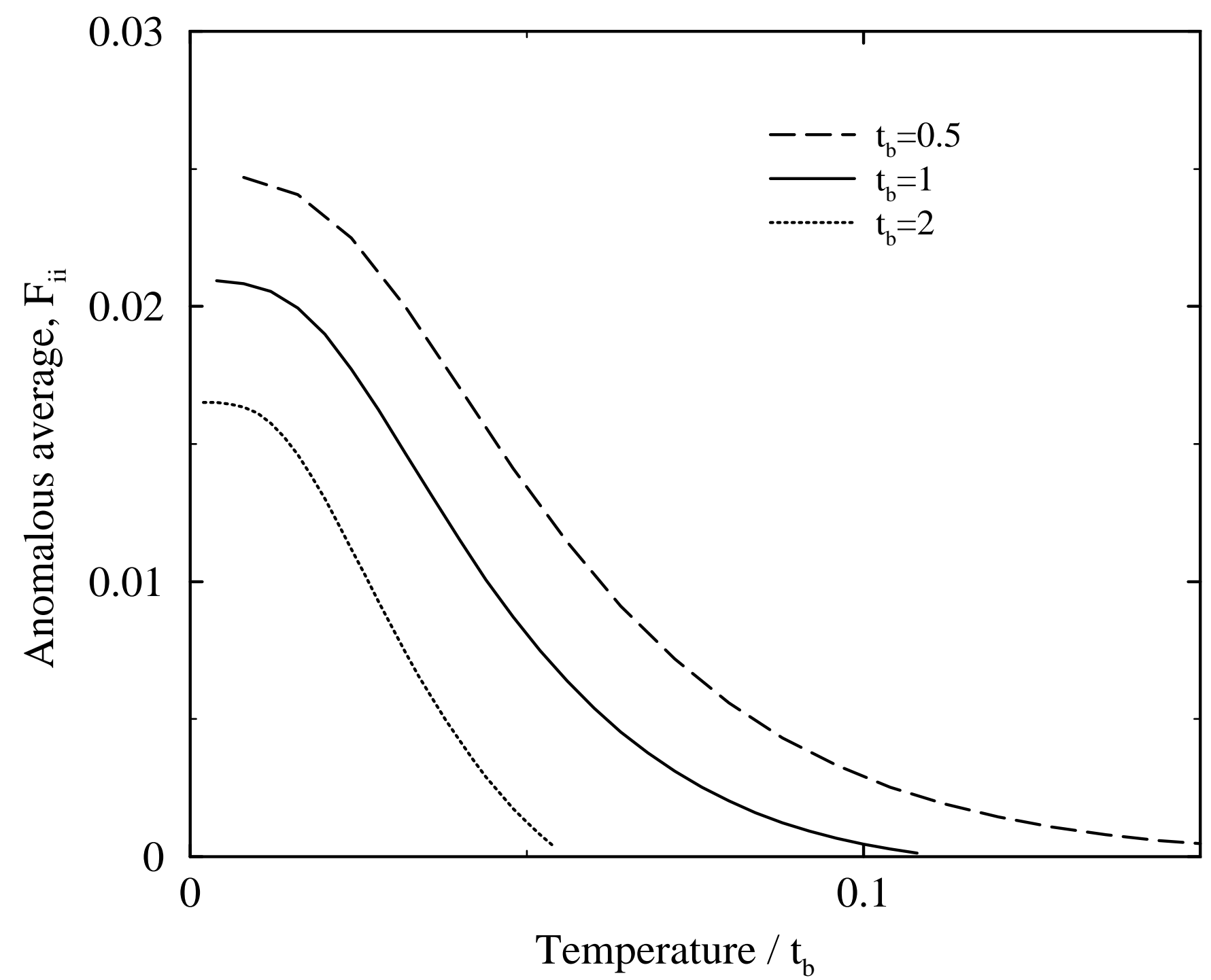

FIG. 10. The variation with temperature of the anomalous average, $F_{i i}=F\left(\mathbf{r}_{i}, \mathbf{r}_{i}, \tau=0^{+}\right)$, for the plane at the center of the barrier region. The different curves are results for different values of the barrier hopping integral, $t_{b}$, with the temperature normalized by $t_{b}$. 


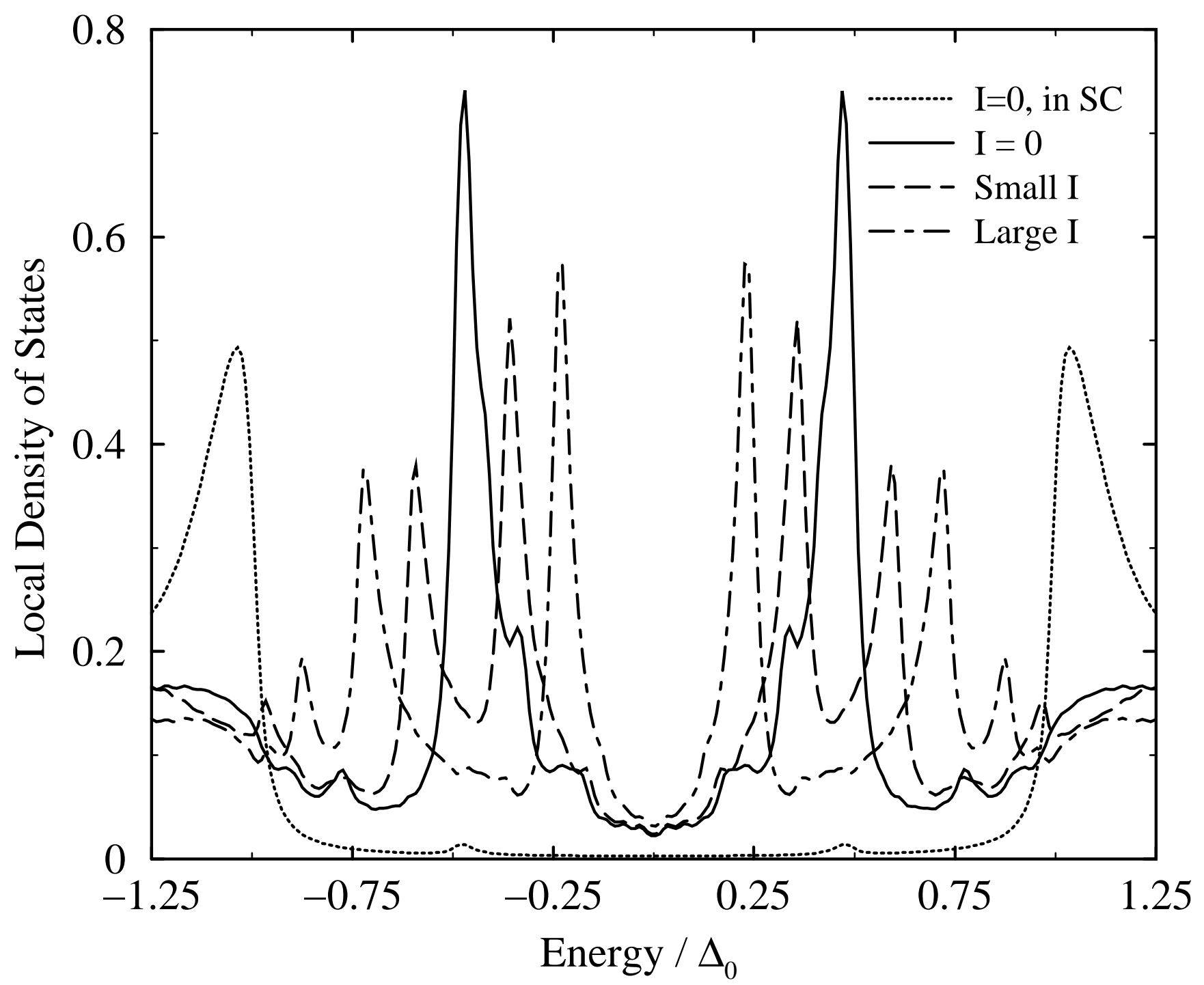

FIG. 11. The local density of states at the center of a Josephson junction (the plane $\alpha=0$ ), with increasing current flow through the device $\left(N_{b}=20\right)$. The solid line is for zero current, the dashed line is for small current, and the chain-dashed line is for large current. The density of states within the superconducting region (plane $\alpha=25$ ) at zero current flow, where $\Delta_{0}=0.198$ is shown in the dotted line as a comparison. Note that the small bump at $E=0.5 \Delta_{0}$ for the superconducting region arises from the self-consistency relation and the proximity of this superconducting plane to the barrier. 


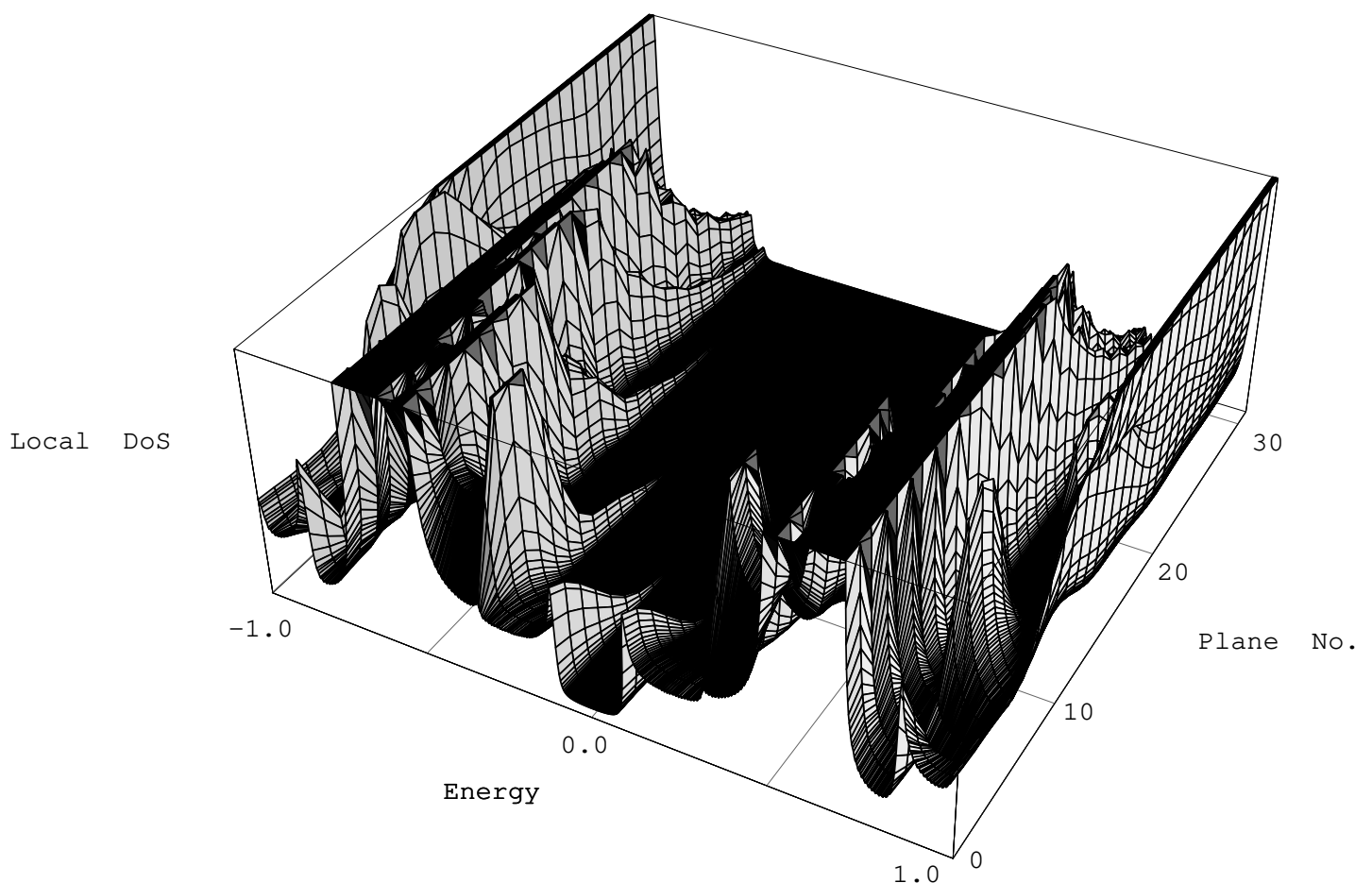


FIG. 12. The evolution of the local density of states within the gap, as a function of position in a Josephson junction. The energy axis is normalized by $\Delta_{0}=0.198$. Note there are even parity bound states, with a maximum at the center plane, and odd parity states with a node at that point. The self-consistent solution is for a barrier of width 10 planes $\left(N_{b}=10\right)$.

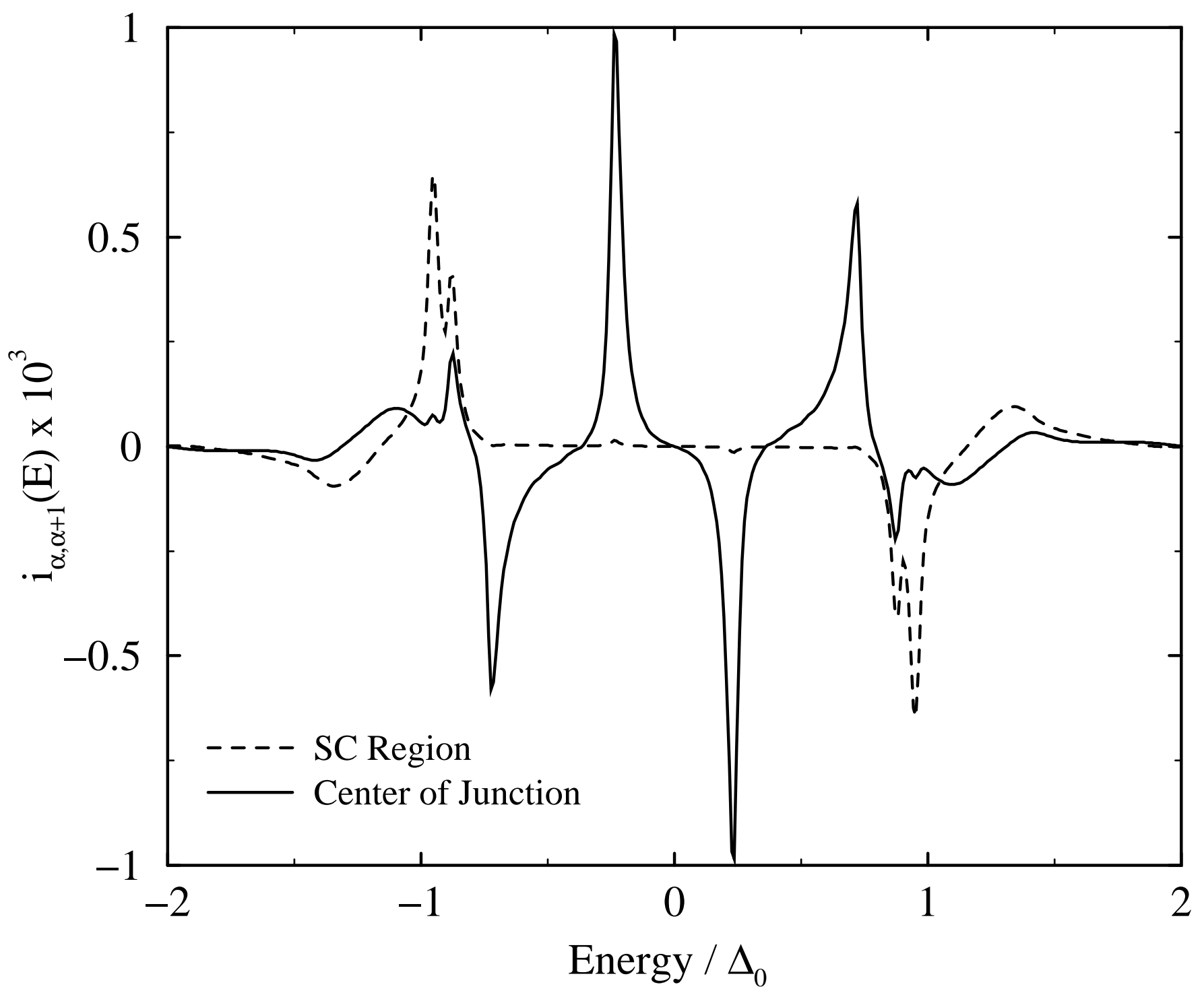

FIG. 13. The local current-carrying density of states, $i_{\alpha, \alpha+1}(E)$ at the center of a Josephson junction (where $\alpha=0$ ) and in the superconducting region (where $\alpha=25$ ). In this junction $N_{b}=20$. 


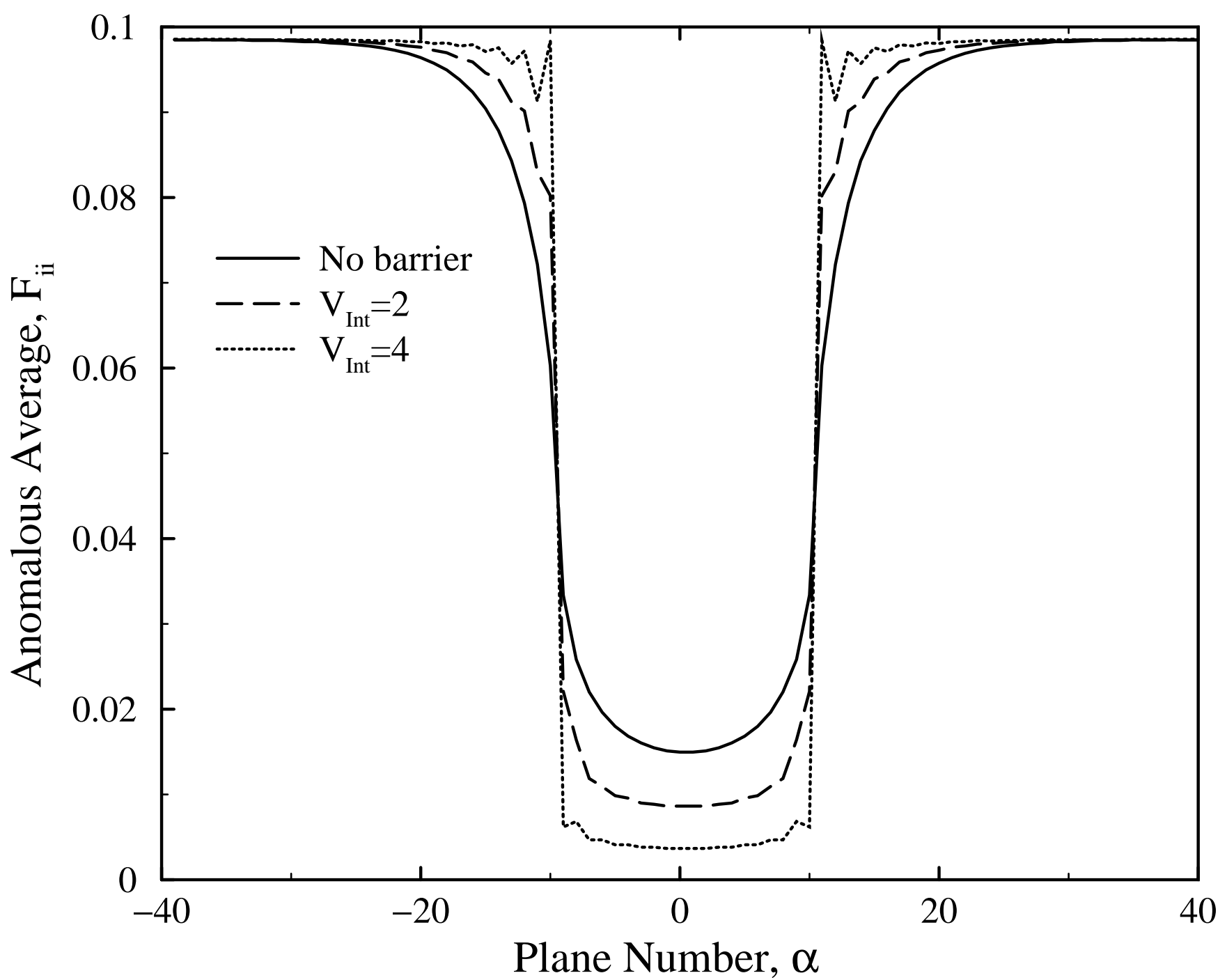

FIG. 14. The off-diagonal local Green's function, $F_{i i}$, indicating Friedel oscillations and a reduced proximity effect as the interfacial scattering is increased. 


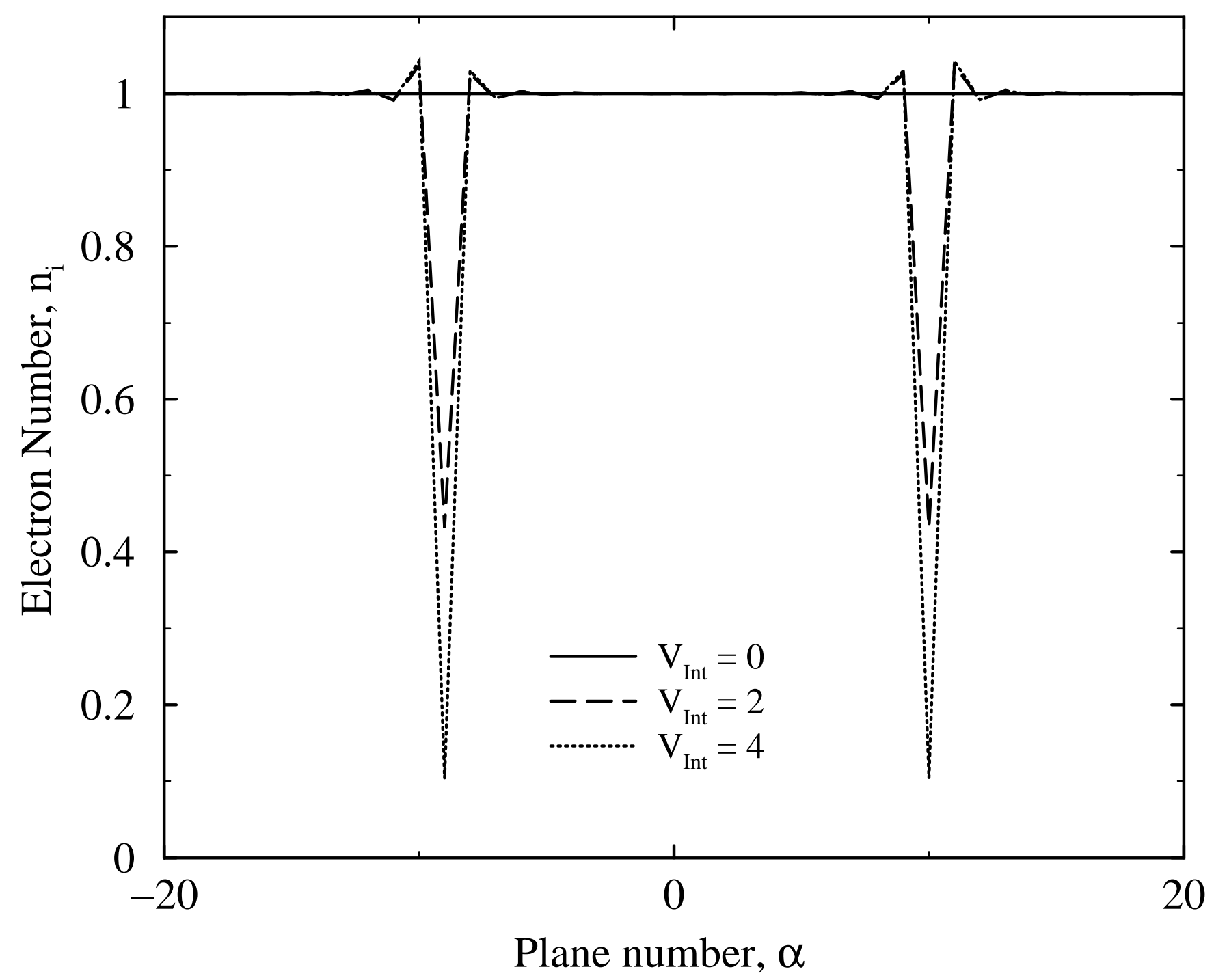

FIG. 15. Friedel oscillations in the electron density due to an interfacial scattering potential. 


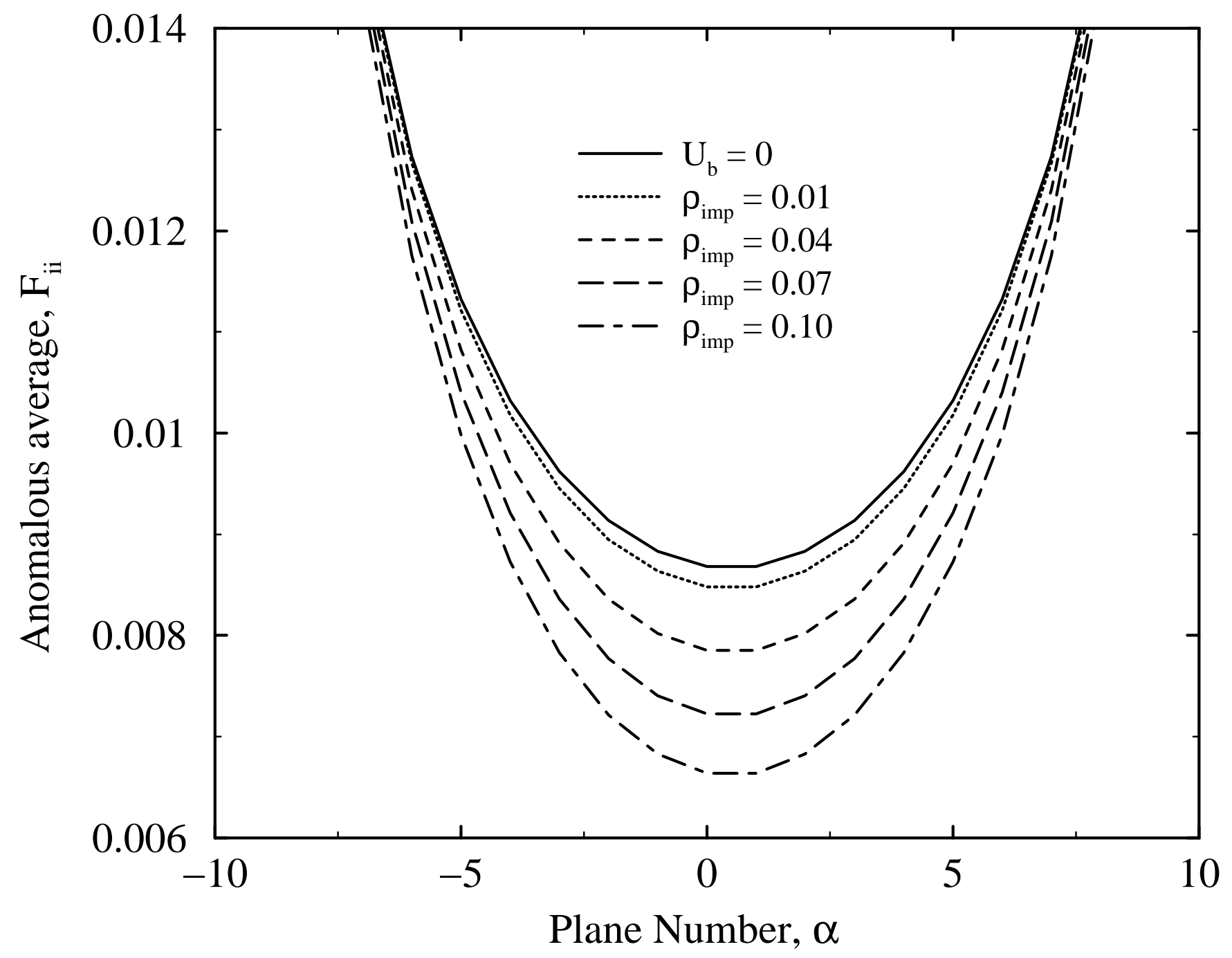

FIG. 16. The reduction of the anomalous average, $F_{i i}=F\left(\mathbf{r}_{i}, \mathbf{r}_{i}, \tau=0^{+}\right)$, for planes near the center of the barrier, with increasing impurity concentration, $\rho_{i}$, and $U^{F K}=-2$. 


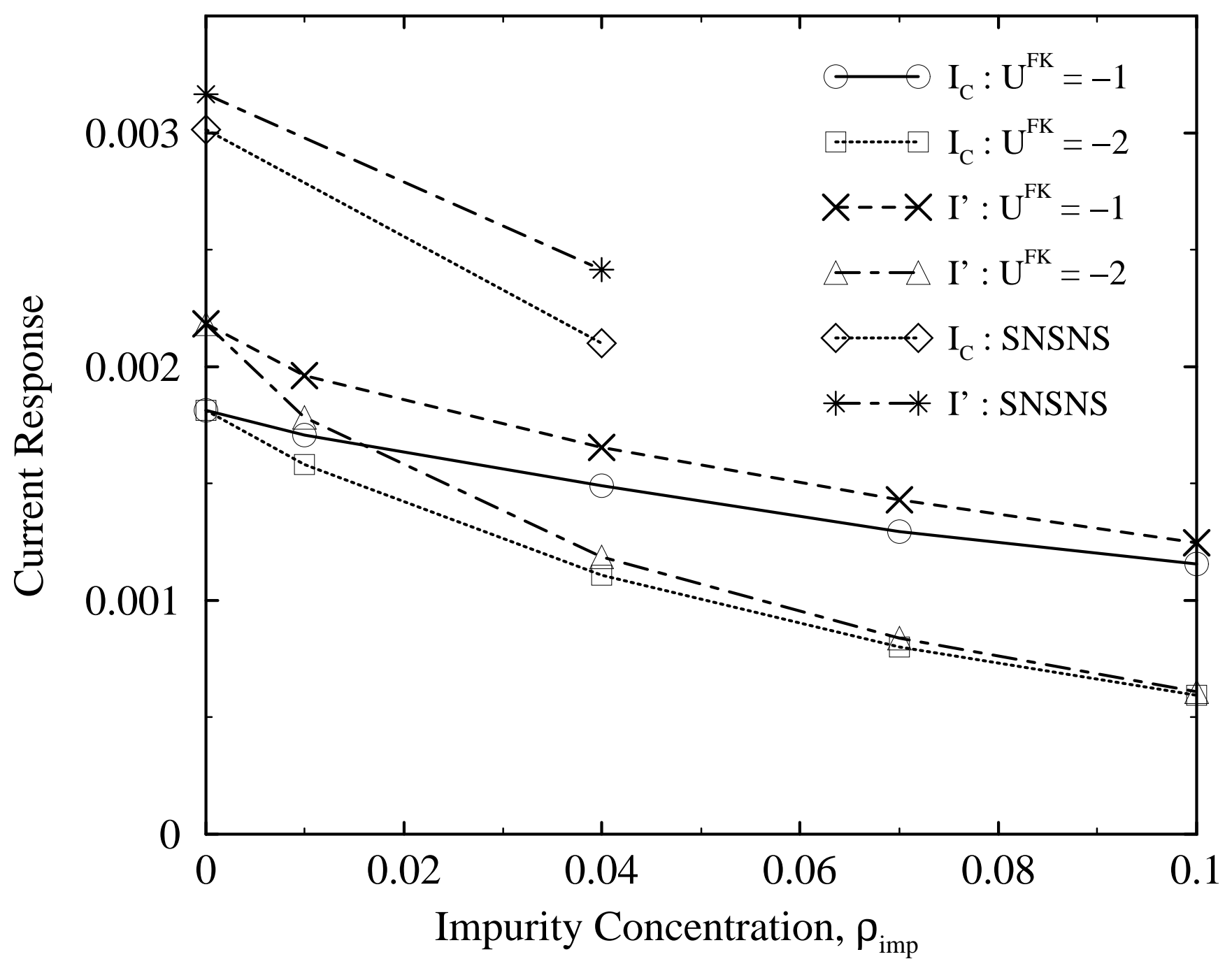

FIG. 17. The critical current, $I_{c}$, and linear response current, $I^{\prime}$, as a function of charge impurities. Note that $I_{c}$ and $I^{\prime}$ decrease as impurities are added to the barrier region. The greater scattering potential of $U^{F K}=-2$ results in greater decrease in current responses, compared to a scattering potential of $U_{F K}=-1$. If superconducting planes are inserted in the middle of the barrier with $U^{F K}=-2$ (SNSNS) the critical current is seen to increase dramatically. 


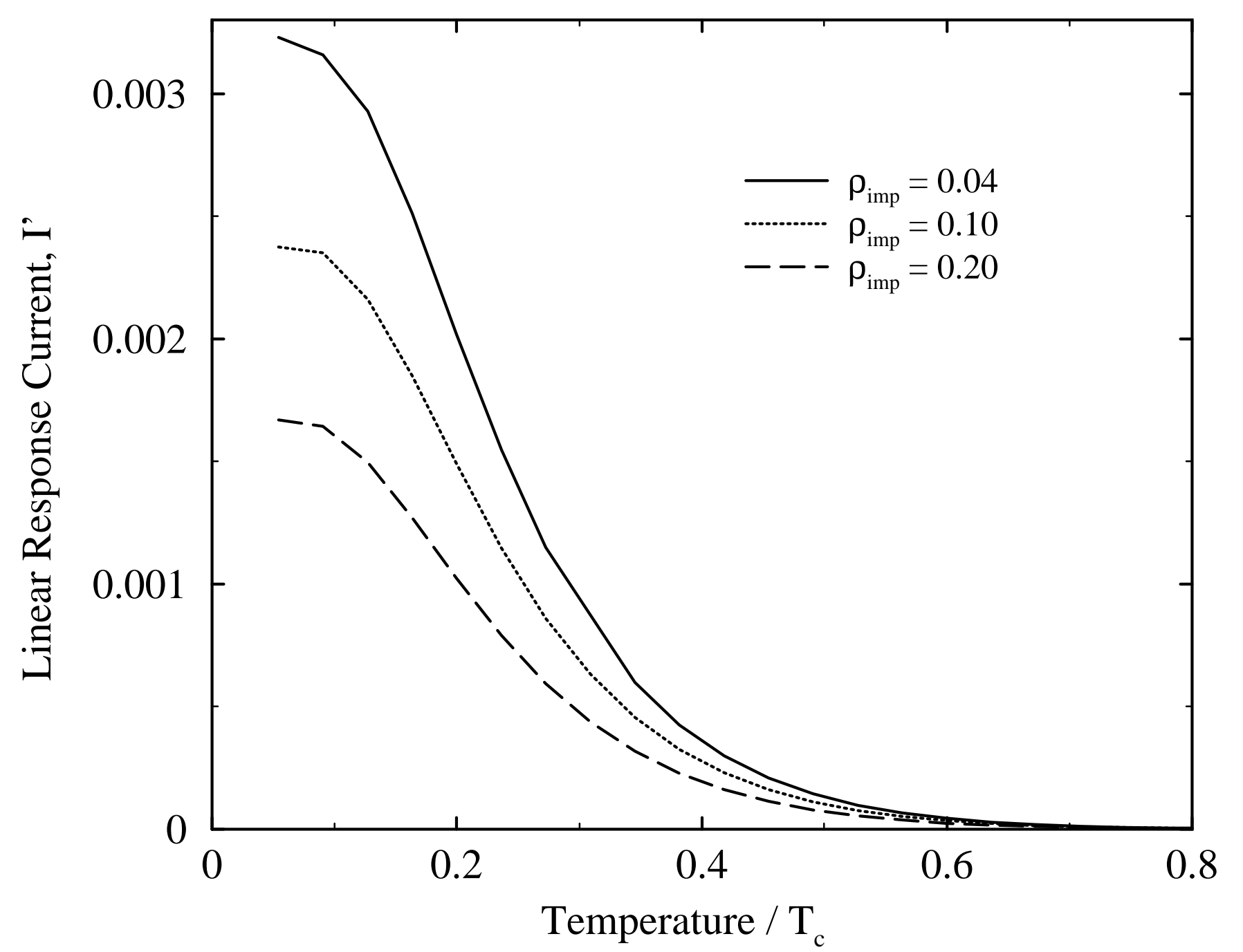

FIG. 18. The linear response current, $I^{\prime}$ as a function of temperature for increasing impurity concentrations in the barrier. 


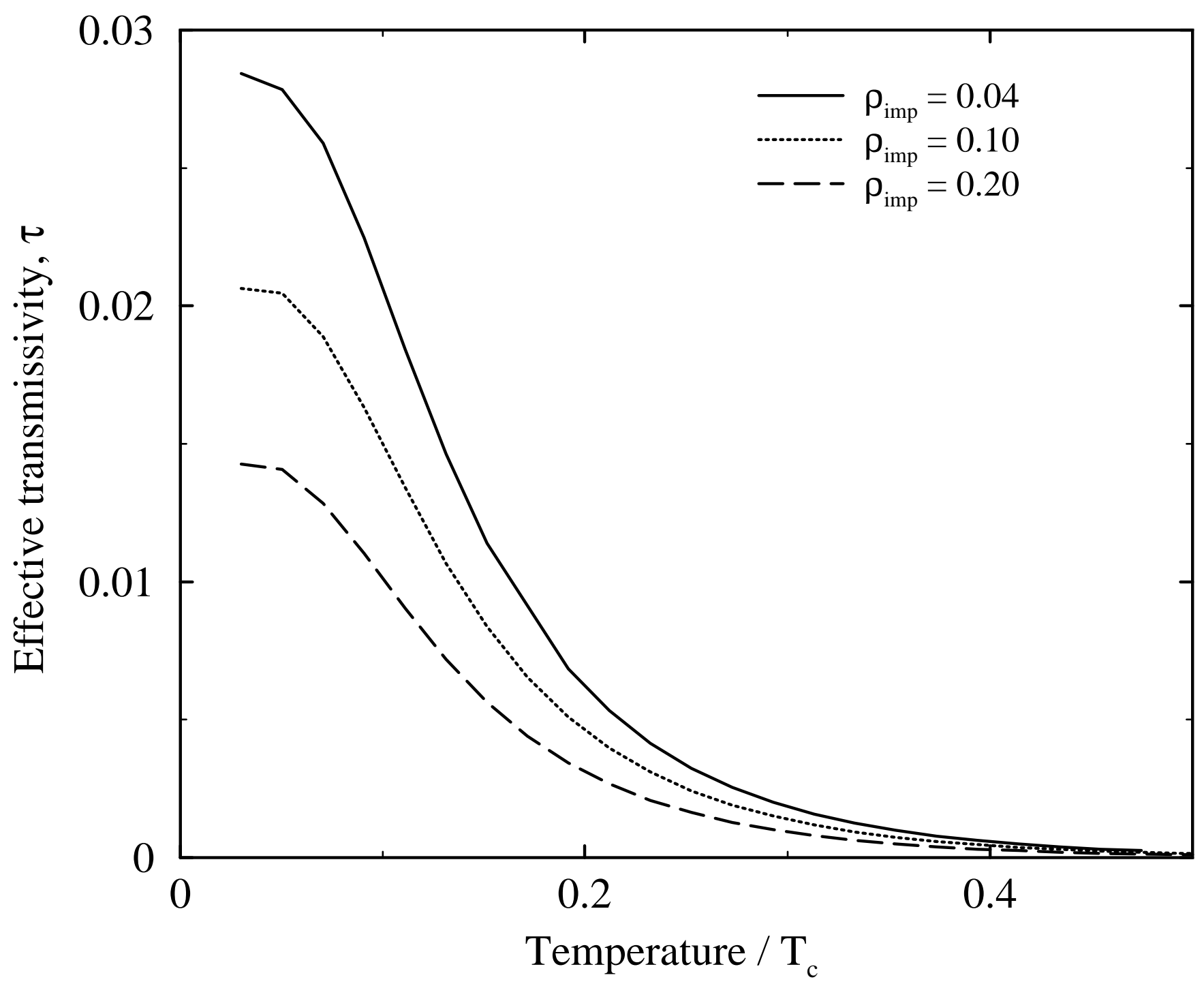

FIG. 19. The effective transmissivity as a function of temperature. Note how the transmissivity decays with increasing temperature, shown here for barriers containing impurity scatterers. 


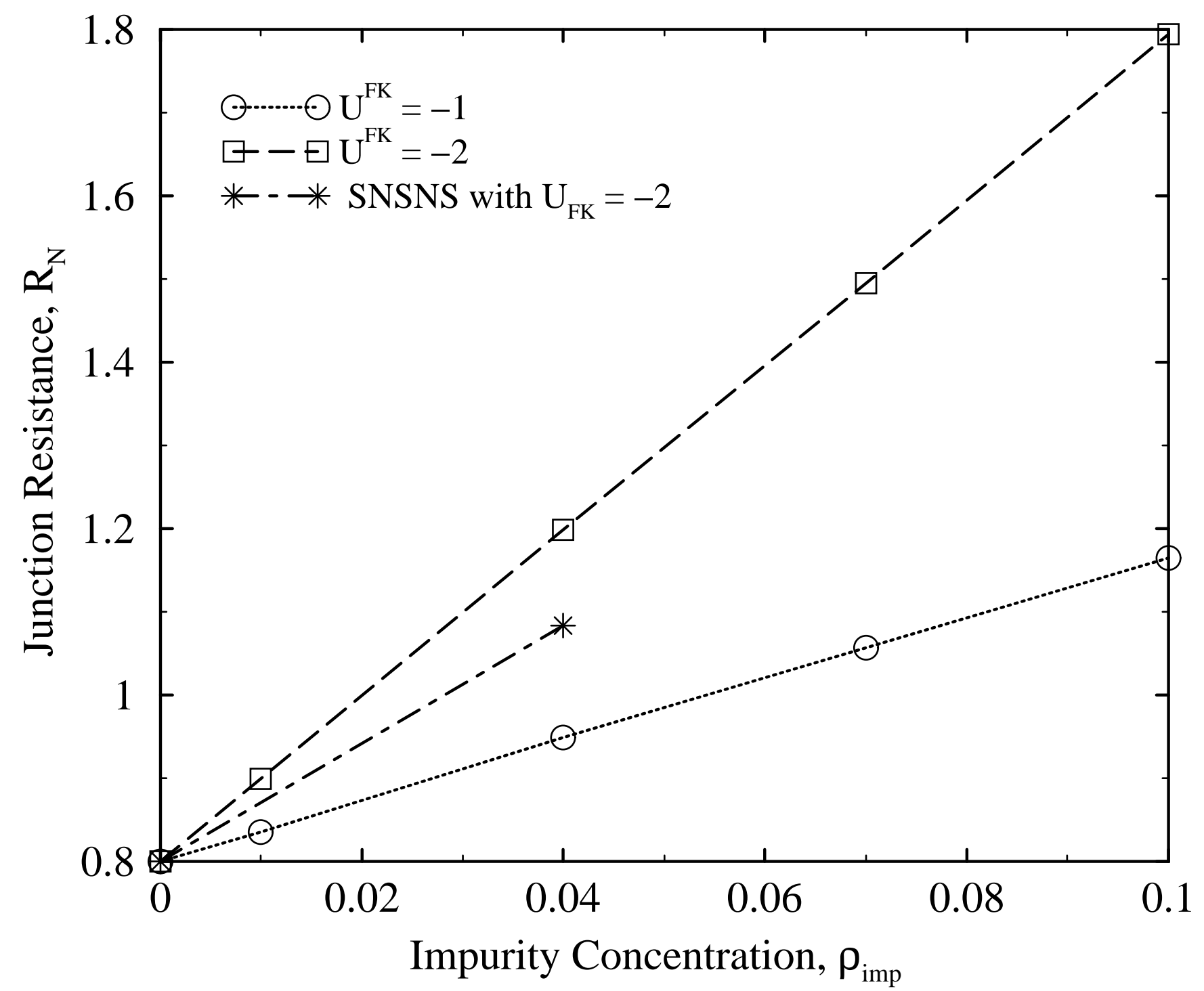

FIG. 20. The normal state resistance of a junction versus impurity concentration in the barrier. The scattering potential is greater, $U^{F K}=-2$ for the higher resistance curve compared to $U^{F K}=-1$ for the lower curve. Insertion of extra planes of superconducting material in the center of the barrier with $U^{F K}=-2(\mathrm{SNSNS})$ reduces the resistance in this case. 


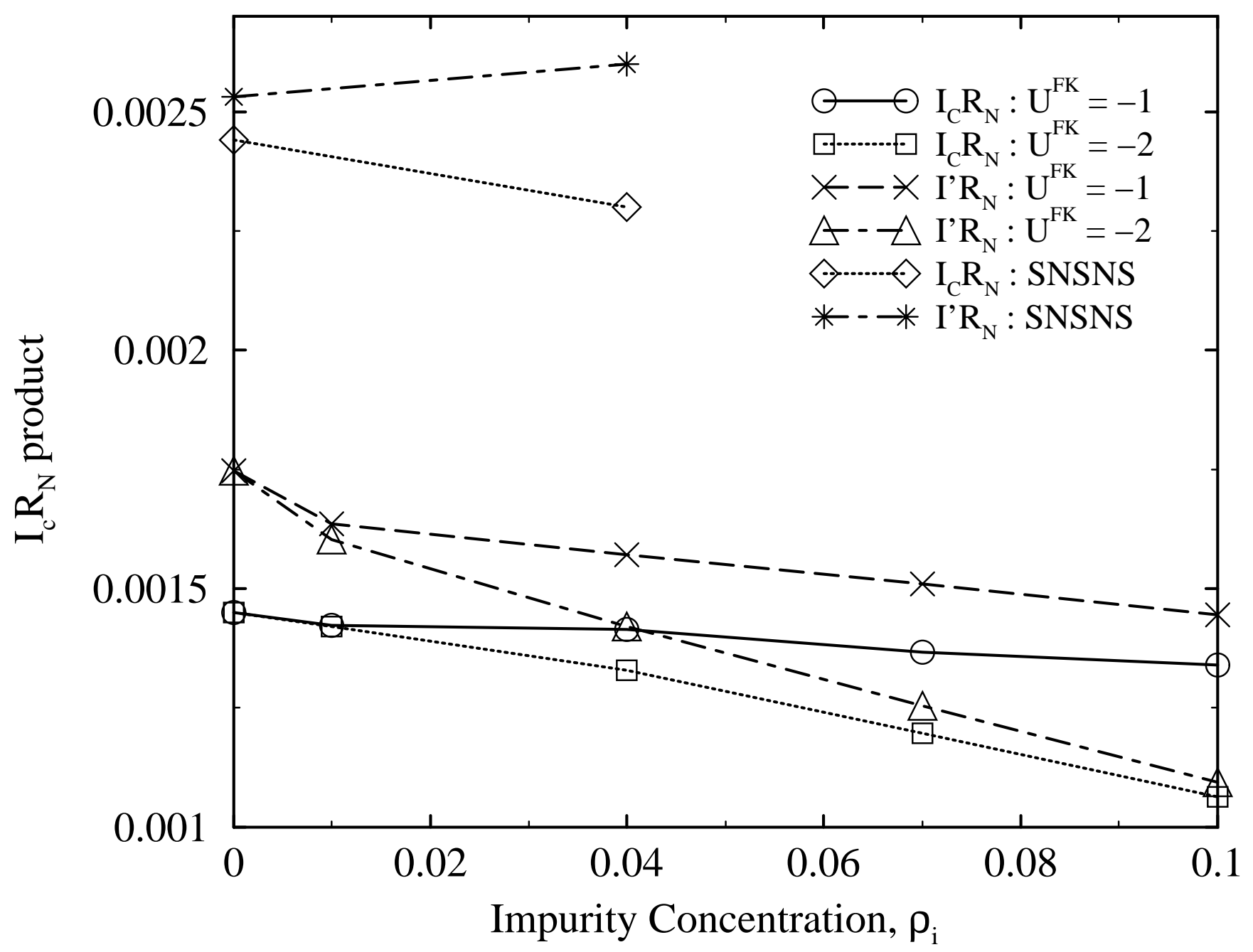

FIG. 21. The product of normal resistance of a junction with critical current, $I_{c}$, and linear response current, $I^{\prime}$, for a junction with impurity scattering in the barrier. Scattering strengths of $U^{F K}=-1$ and $U^{F K}=-2$ are plotted. Increased figures of merit occur for both clean systems and for the SNSNS structures. 